Accepted refereed manuscript of: Aguirre-Gutiérrez J, Rifai S, Shenkin A, Oliveras I, Bentley LP, Svátek M, Girardin CAJ, Both S, Riutta T, Berenguer E, Kissling WD, Bauman D, Abernethy K, Jeffery KJ \& White LJT (2021) Pantropical modelling of canopy functional traits using Sentinel-2 remote sensing data. Remote Sensing of Environment, 252, Art. No.: 112122. https://doi.org/10.1016/j.rse.2020.112122

(C) 2020, Elsevier. Licensed under the Creative Commons Attribution-NonCommercial-NoDerivatives 4.0 International http://creativecommons.org/licenses/by-nc-nd/4.0/

\title{
Pantropical modelling of canopy functional traits using Sentinel-2 remote sensing data
}

Jesús Aguirre-Gutiérrez et al.

Supplementary information 

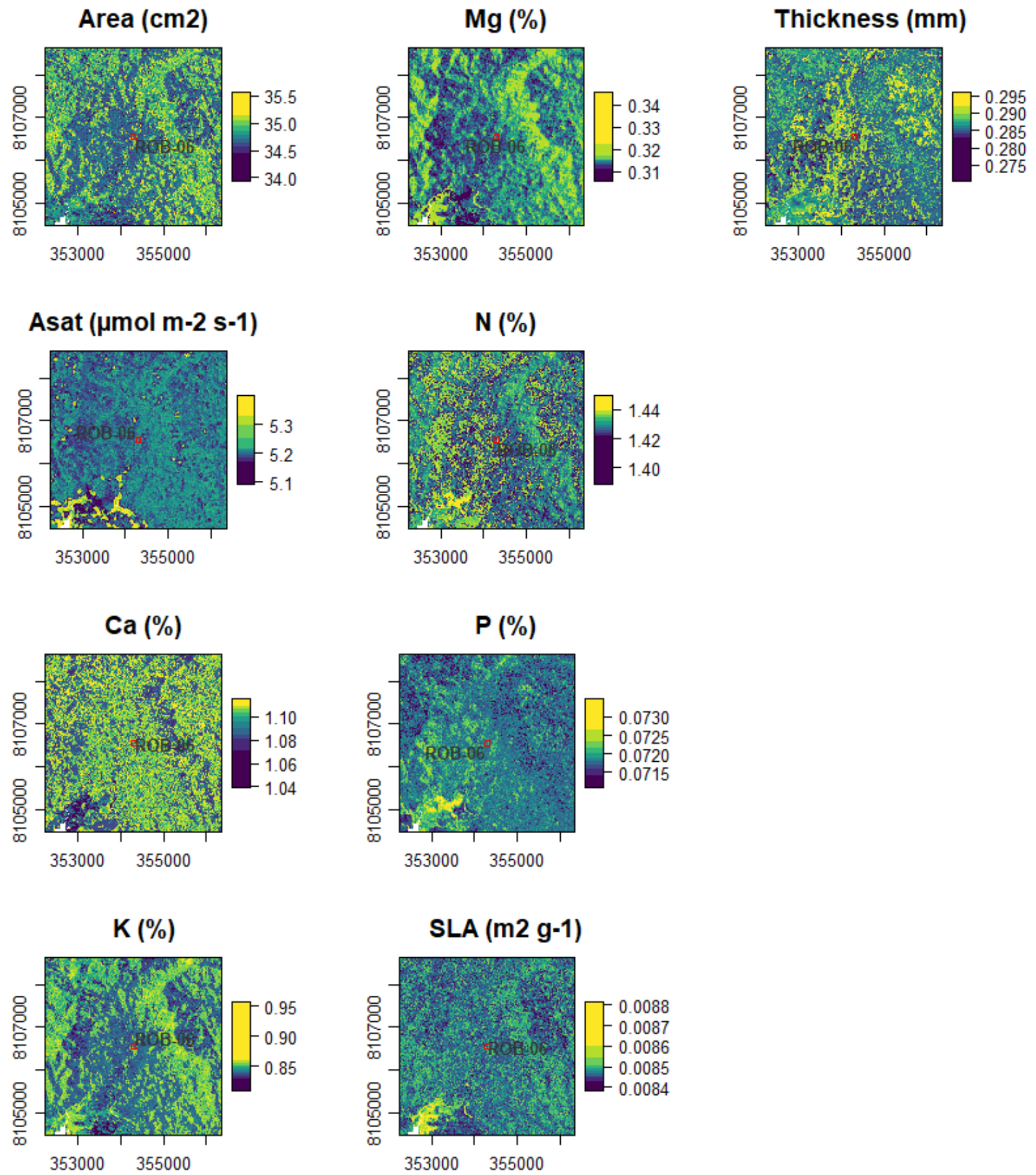

Figure S1. Spatial predictions of trait distributions for plant traits in Australia following the general model and predicting at $10 \times 10 \mathrm{~m}$ pixel resolution. Predictions are only shown for an area surrounding one of the focus vegetation plots. 

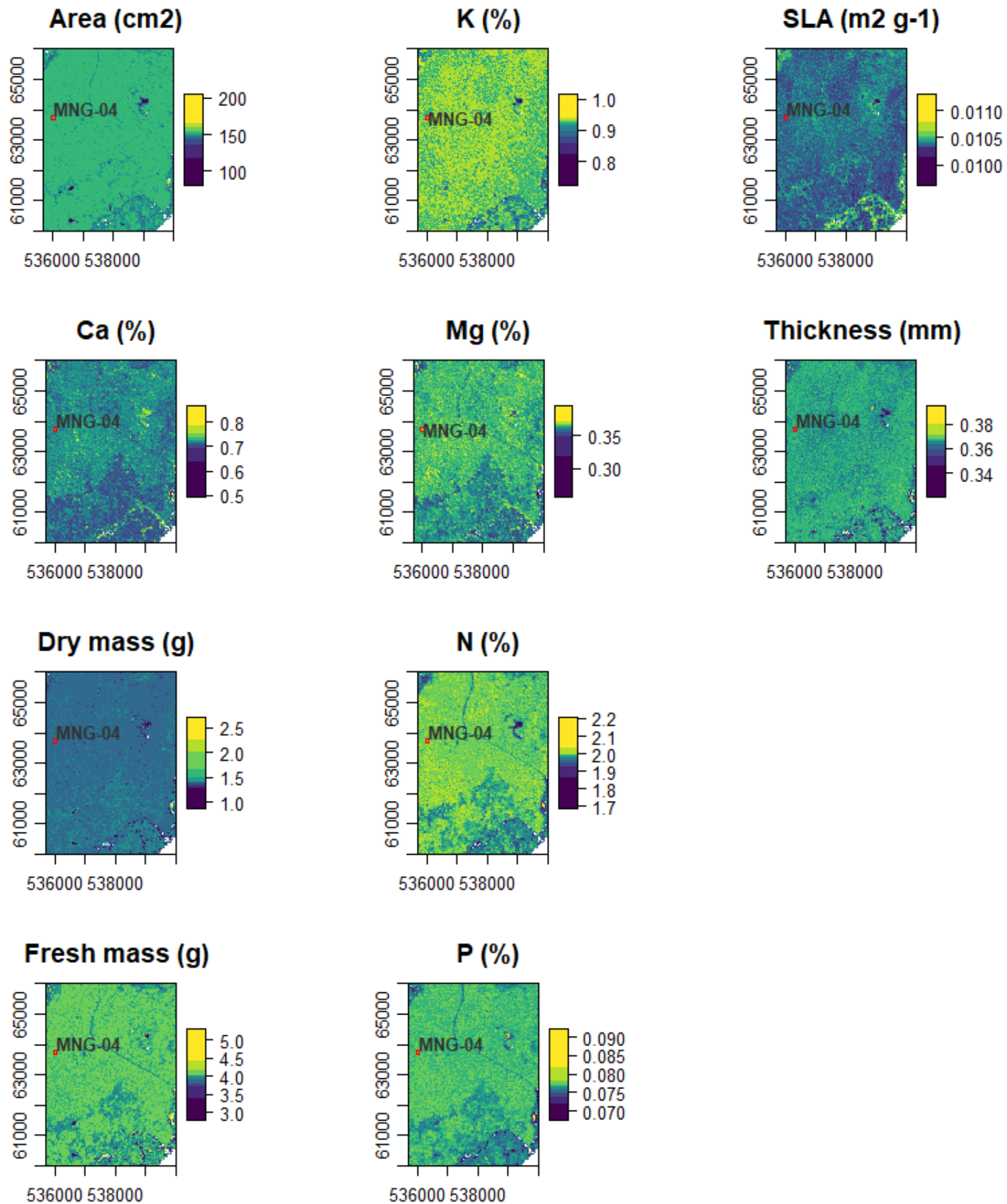

Figure S2. Spatial predictions of trait distributions for plant traits in Gabon following the general model and predicting at $10 \times 10 \mathrm{~m}$ pixel resolution. Predictions are only shown for an area surrounding one of the focus vegetation plots. 

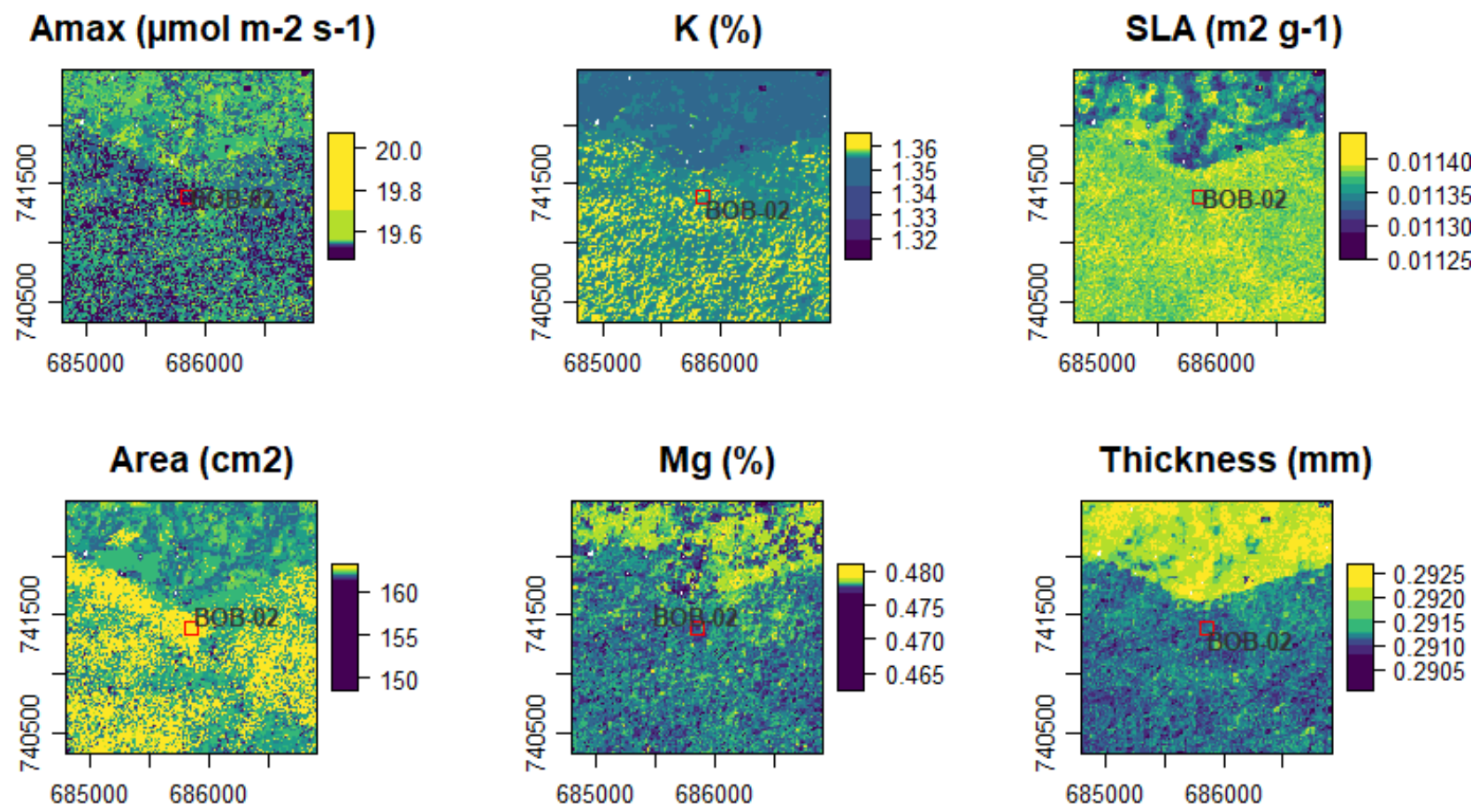

\section{Asat ( $\mu \mathrm{mol} \mathrm{m}-2 \mathrm{~s}-1)$}
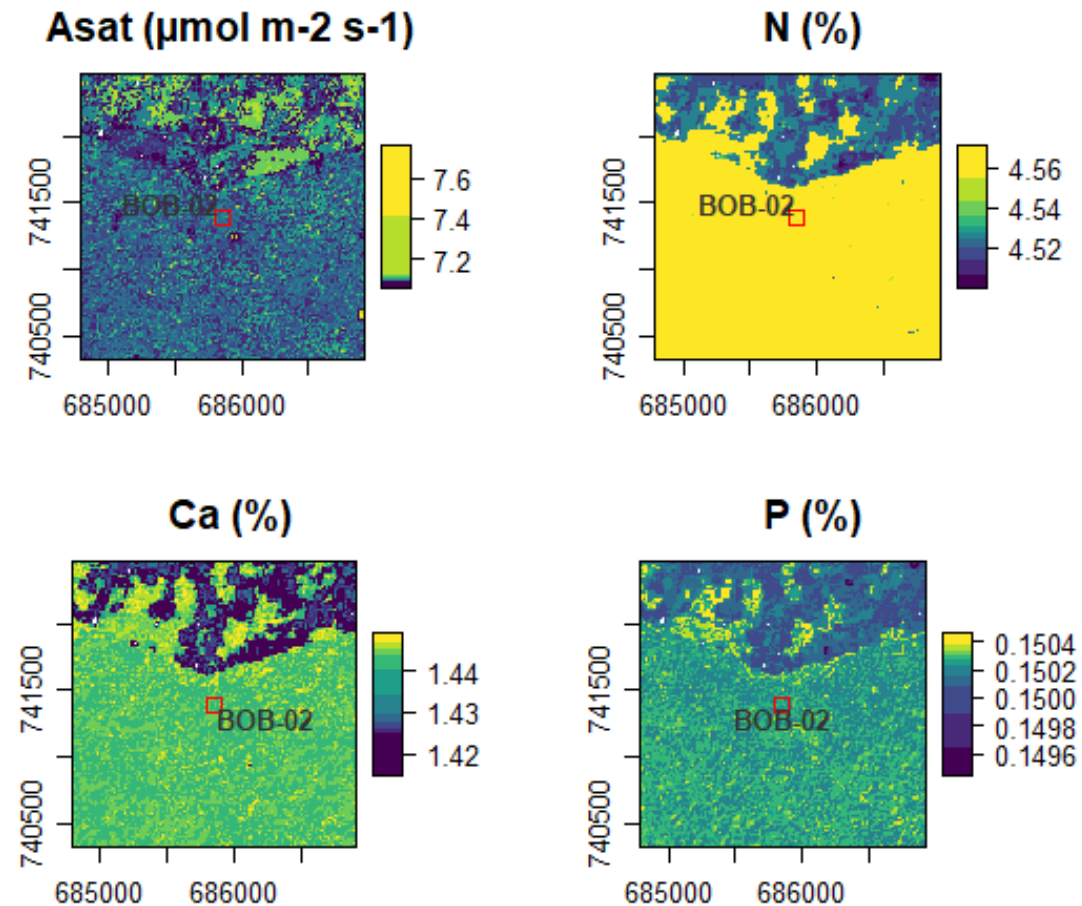

Figure S3. Spatial predictions of trait distributions for plant traits in Ghana following the general model and predicting at $10 \times \mathbf{1 0 m}$ pixel resolution. Predictions are only shown for an area surrounding one of the focus vegetation plots. 

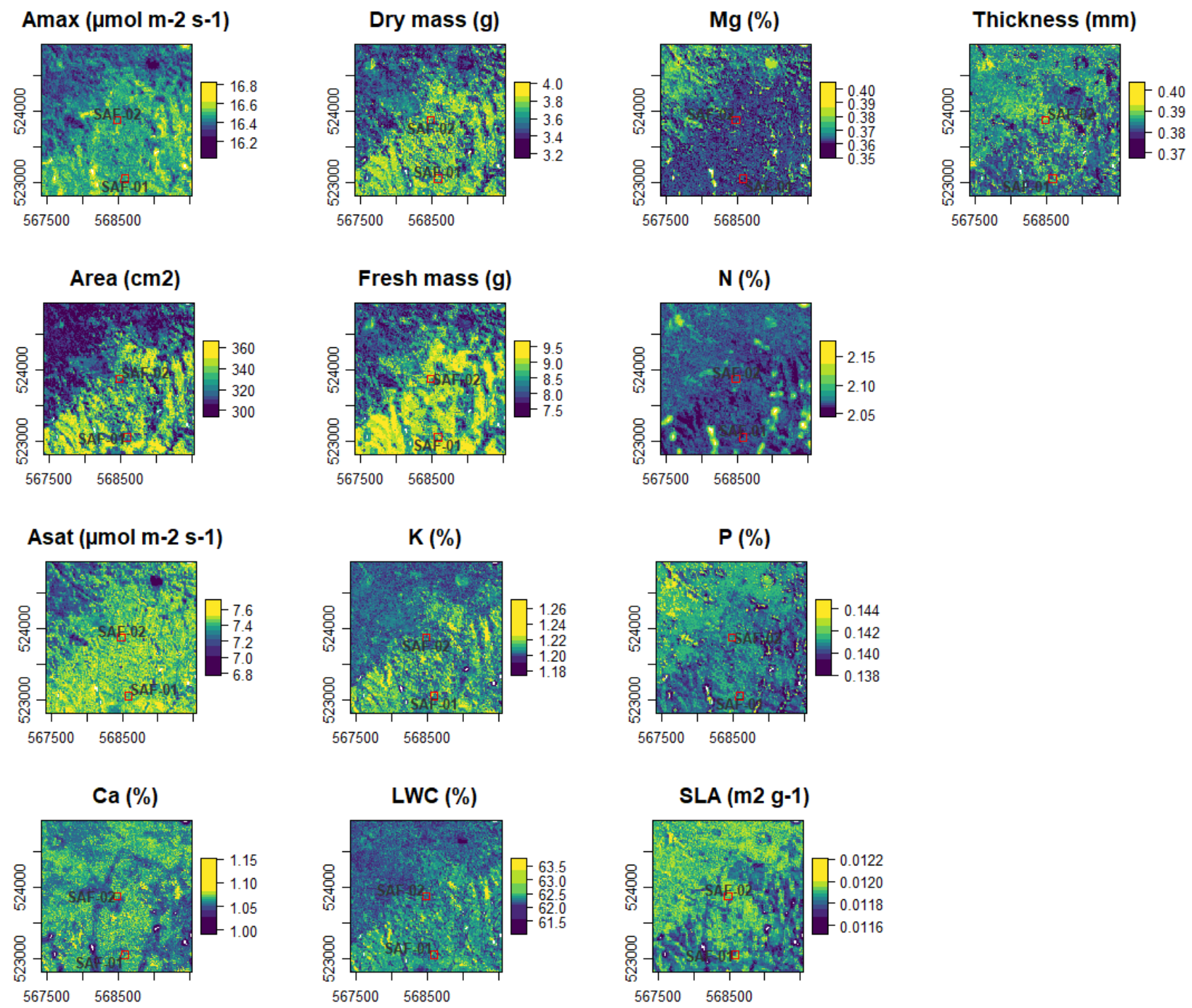

Figure S4. Spatial predictions of trait distributions for plant traits in Malaysia following the general model and predicting at $10 \times 10 \mathrm{~m}$ pixel resolution. Predictions are only shown for an area surrounding two of the focus vegetation plots. 

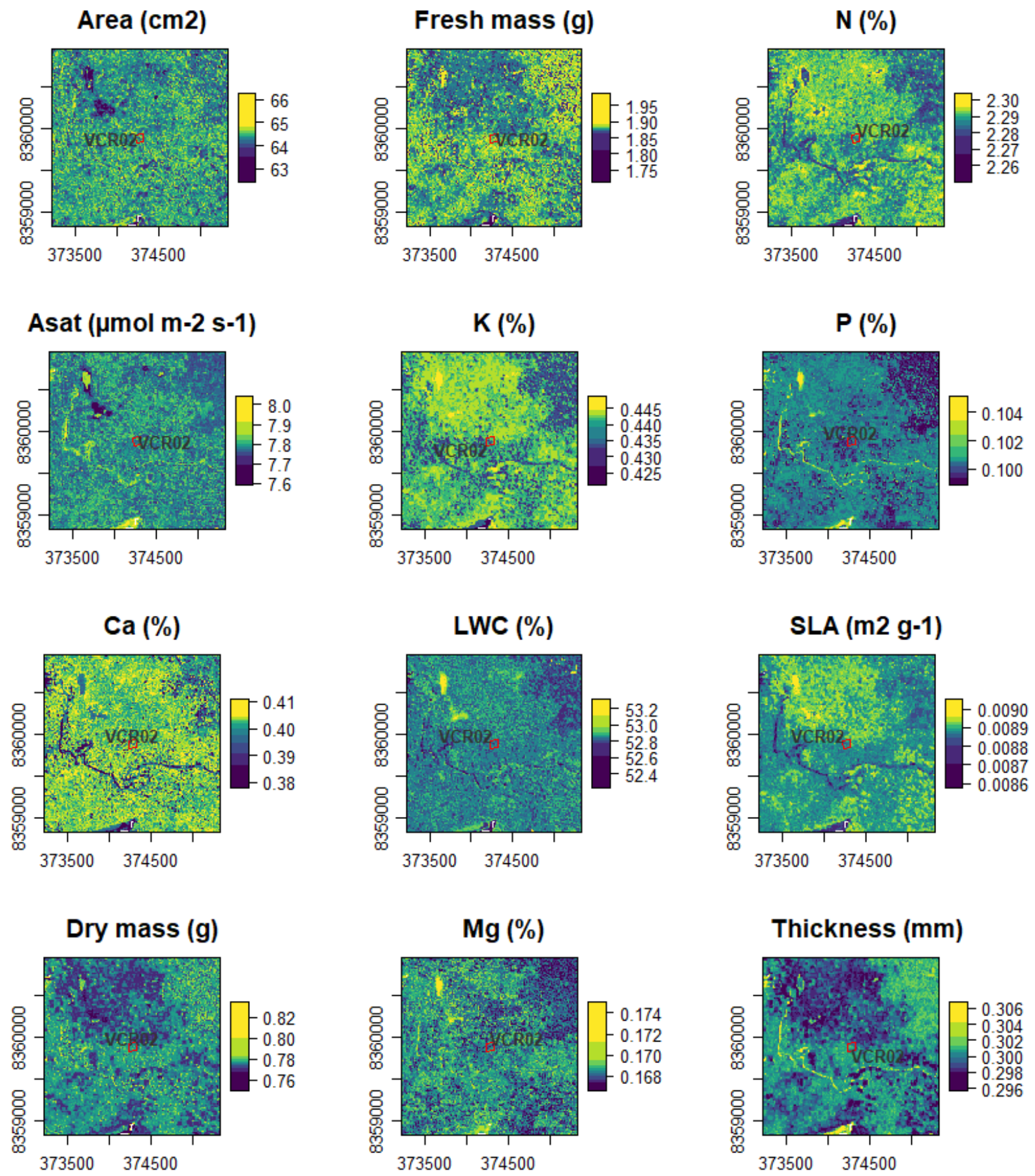

Thickness $(\mathrm{mm})$

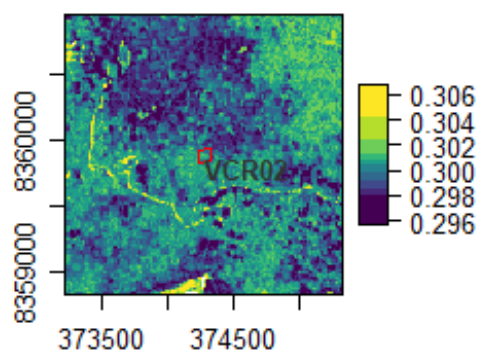

Figure S5. Spatial predictions of trait distributions for plant traits in Brazil -NX (Nova Xavantina) following the general model and predicting at $10 \times 10 \mathrm{~m}$ pixel resolution. Predictions are only shown for an area surrounding one of the focus vegetation plots. 

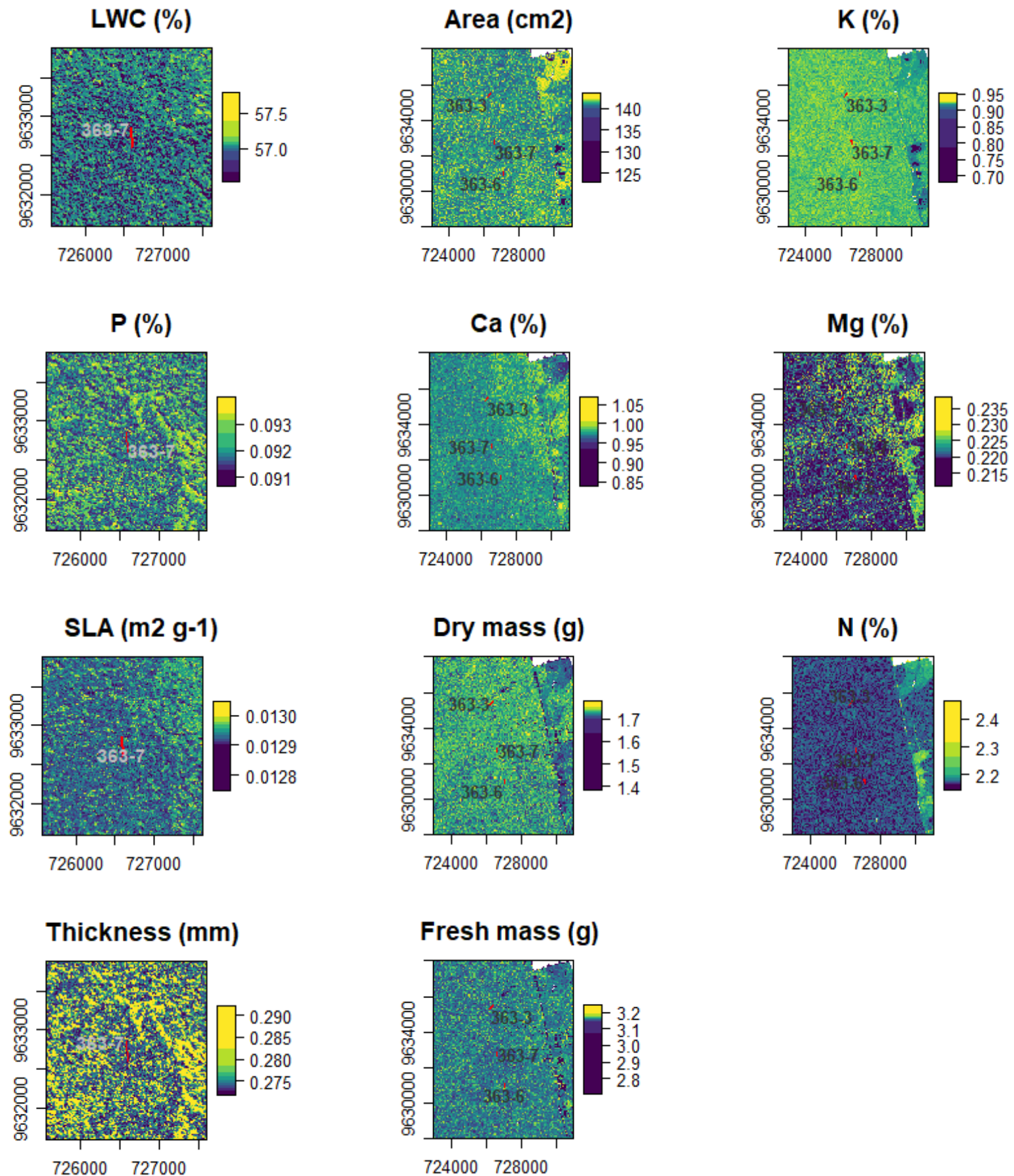

Figure S6. Spatial predictions of trait distributions for plant traits in Brazil -ST (Santarem) following the general model and predicting at $10 \times 10 \mathrm{~m}$ pixel resolution. Predictions are only shown for an area surrounding three of the focus vegetation plots. 

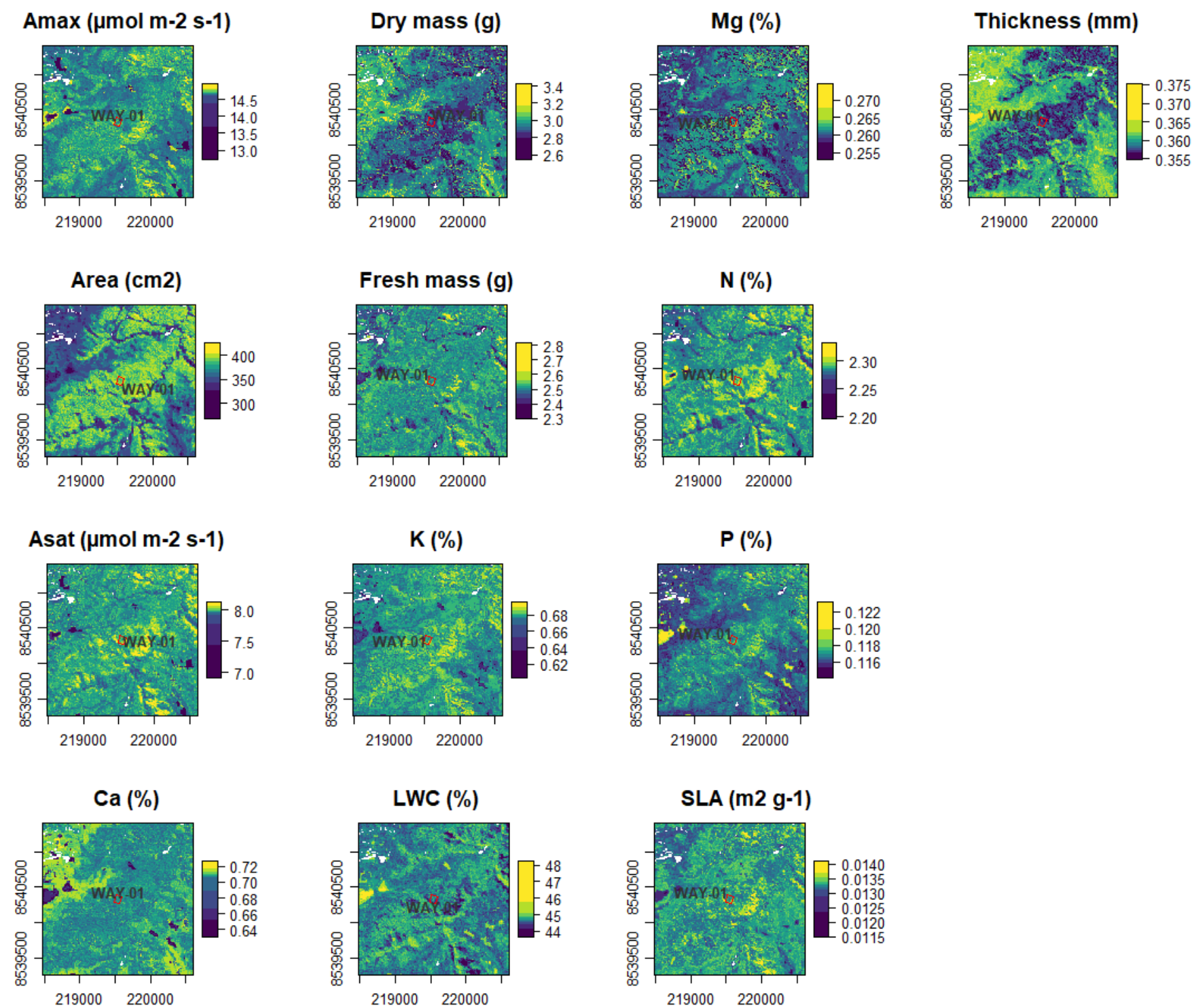

Figure S7. Spatial predictions of trait distributions for plant traits in Peru following the general model and predicting at $10 \times 10 \mathrm{~m}$ pixel resolution. Predictions are only shown for an area surrounding one of the focus vegetation plots. 

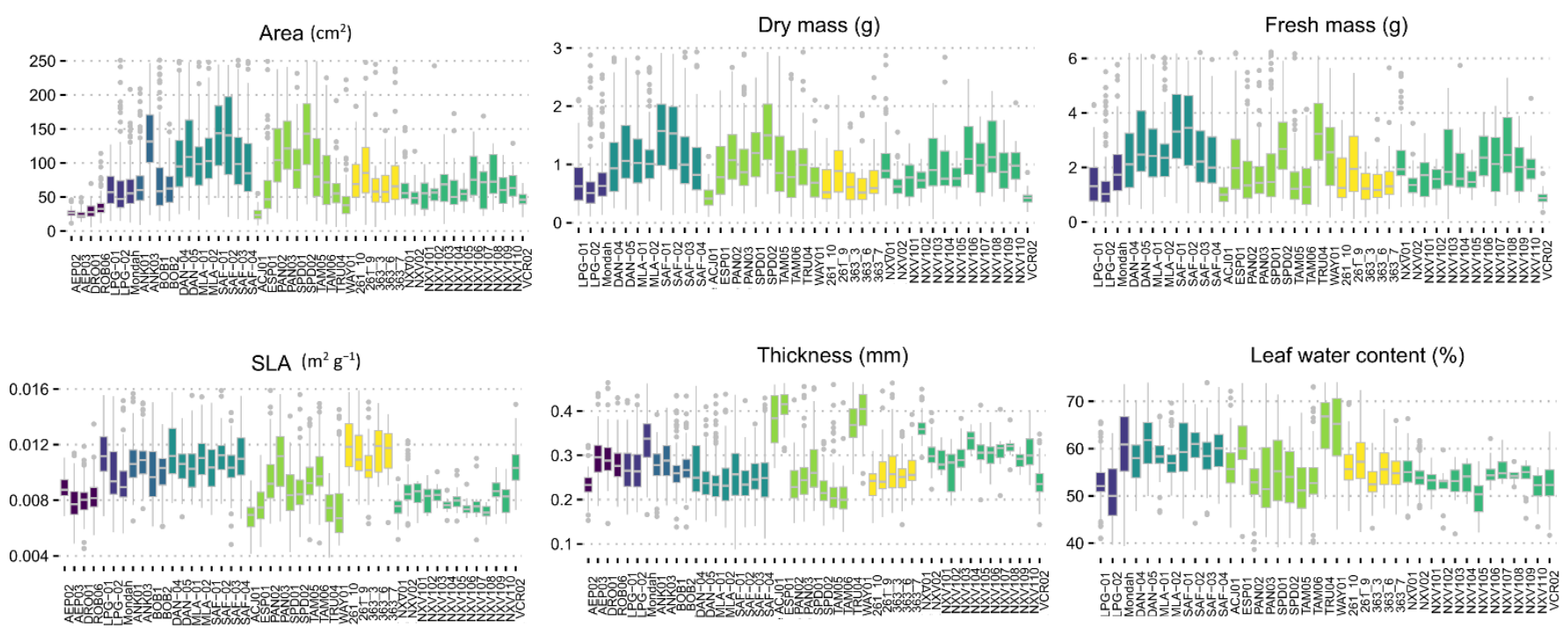

$\mathrm{Ca}(\%)$

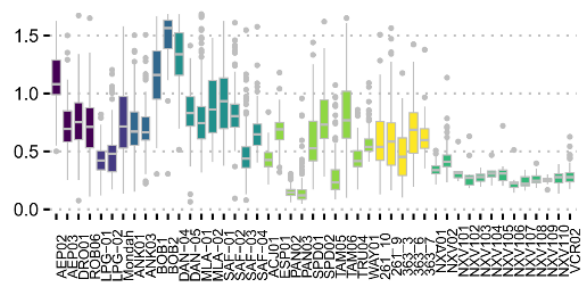

$\mathrm{Mg}(\%)$

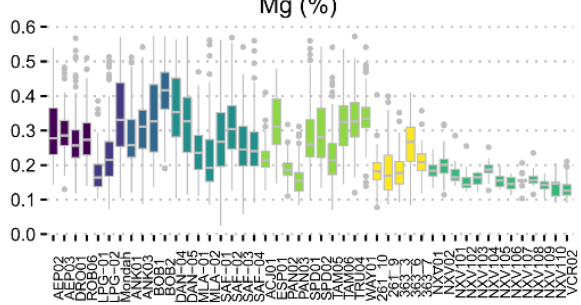

C (\%)

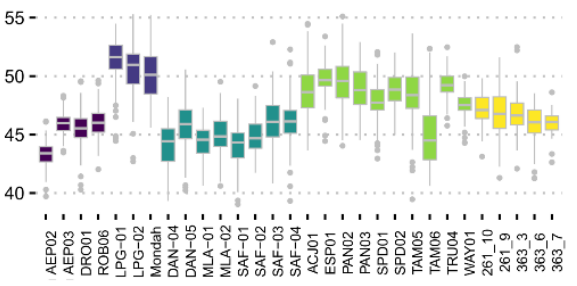

$\mathrm{K}(\%)$

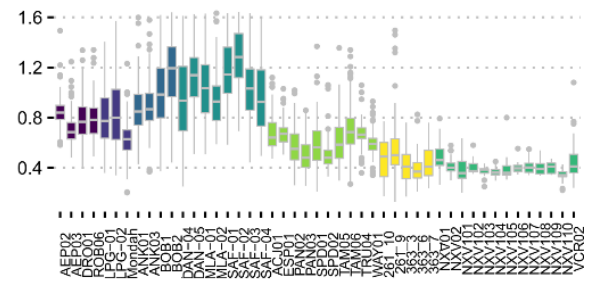

P (\%)

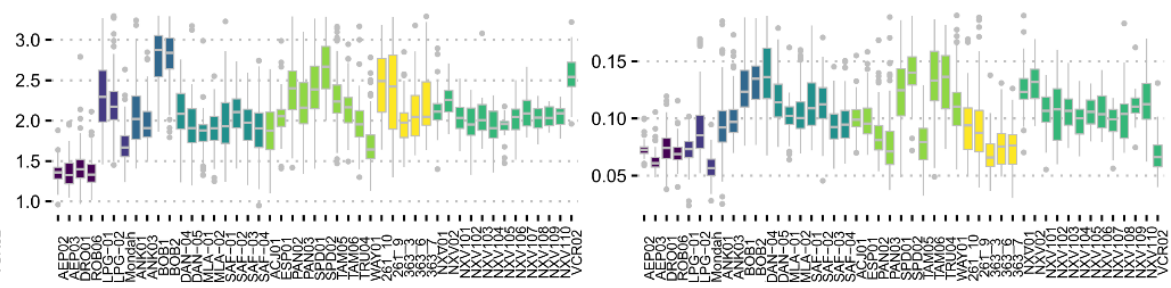

Amax $\left(\mu \mathrm{mol} \mathrm{m} \mathrm{m}^{-2} \mathrm{~s}^{-1}\right)$

Asat $\left(\mu \mathrm{mol} \mathrm{m} \mathrm{m}^{-2} \mathrm{~s}^{-1}\right)$
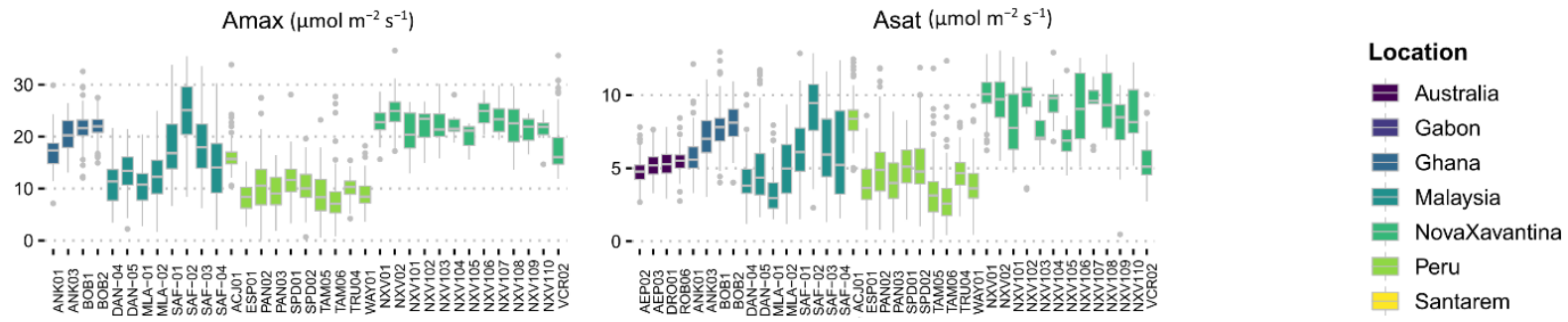

Figure S8. Comparison of trait distributions at the plot level and between tropical regions. For some locations information for all traits was not available. For full details on mean trait value differences among locations see Table S4. 

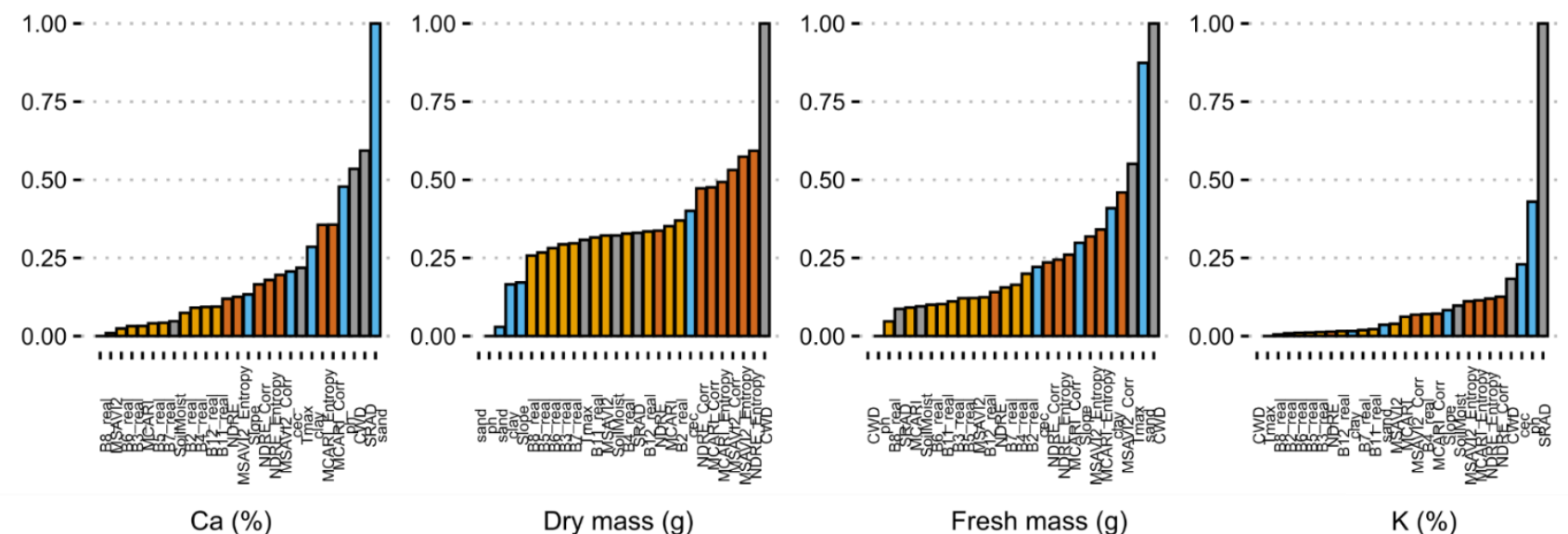

Dry mass (g)

Fresh mass (g)

K (\%)
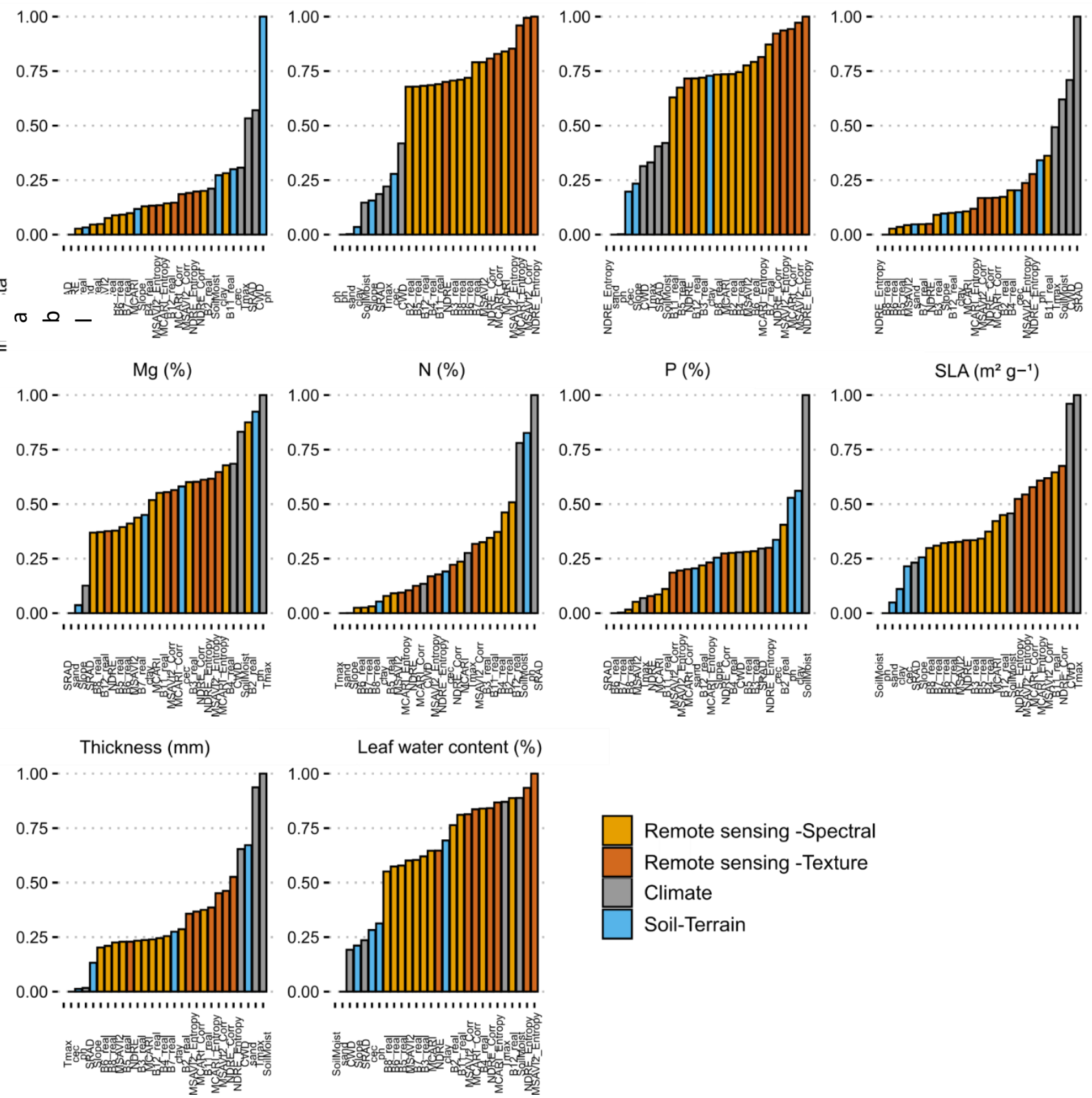

Figure S9. Standardised variable importance of spectral remote sensing, environmental and soil related variables for determining functional trait predictions in the global model. The spectral group contains the select raw bands from the Sentinel- 2 and the vegetation indices; 
Texture contains the Correlation and Entropy metrics from the grey level co-occurrence matrix obtained from the vegetation indices; Climate contains all climatic variables; Soil-Terrain contains all soil characteristics and slope. All variables are described in Table 3.

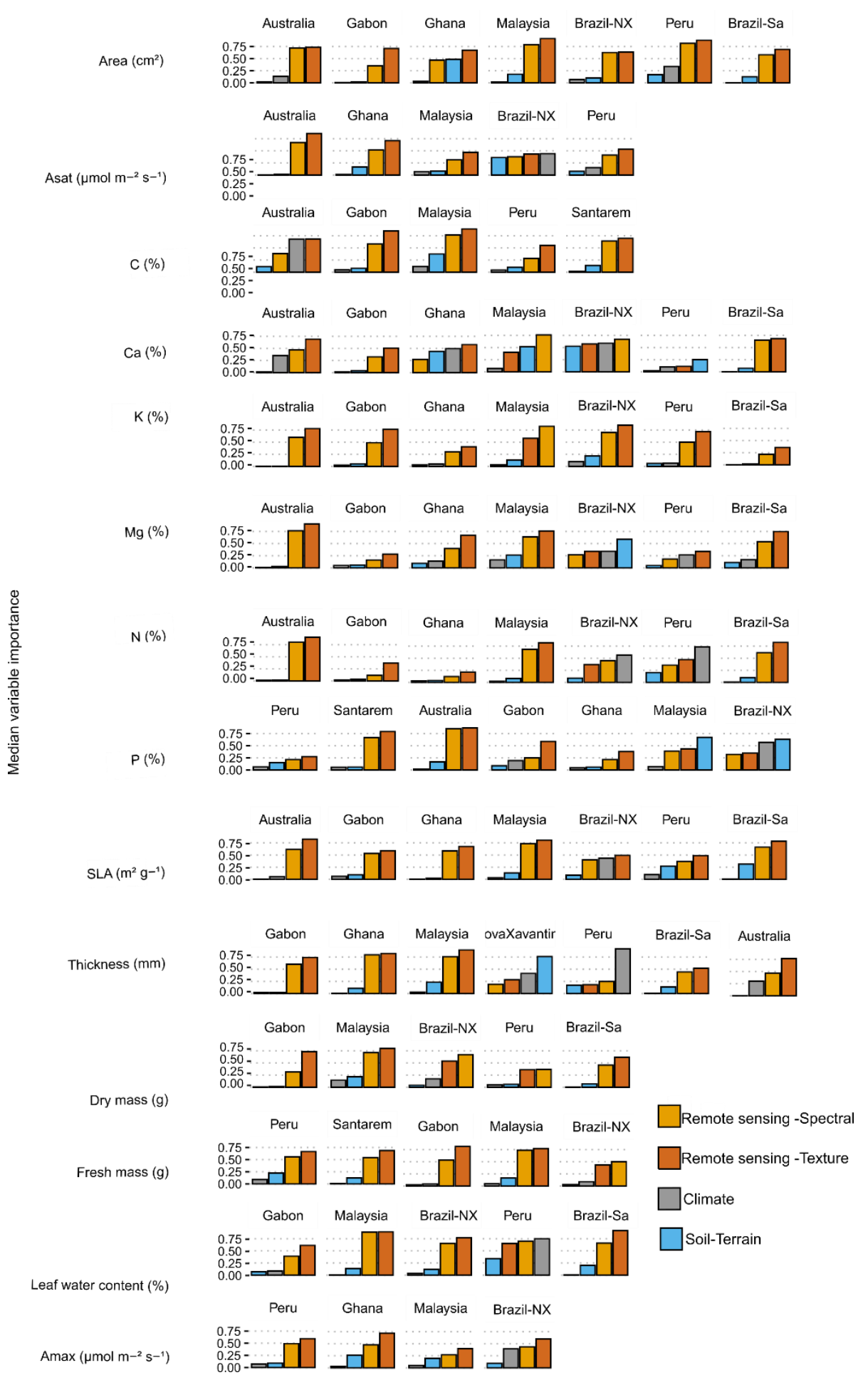

Figure S10. Group averaged variable importance of spectral remote sensing, environmental and soil related variables for determining functional trait predictions in the regional models. The spectral group contains the selected raw bands from the Sentinel-2 and the vegetation indices; Texture contains the Correlation and Entropy metrics from the grey level co-occurrence 
matrix obtained from the vegetation indices; Climate contains all climatic variables; Soil-Terrain contains all soil characteristics and slope. All variables are described in Table 3. 


\section{Full traits collection protocol}

Photosynthetic related traits: Selected branches were immediately recut under water and leaf gas exchange measurements undertaken as soon as possible (within 1-2 hours). Photosynthetic capacity (light-saturated net assimilation rate) was measured at both saturating $\mathrm{CO}_{2}$ concentration $\left(2000 \mathrm{ppm} \mathrm{CO}_{2}\right.$; $\left.\mathrm{A}_{\text {max }}\right)$, and at ambient $\mathrm{CO}_{2}$ concentration $\left(400 \mathrm{ppm} \mathrm{CO}_{2} ; \mathrm{A}_{\text {sat }}\right)$ under saturating light conditions and at a temperature of $25^{\circ} \mathrm{C}$ using a LICOR $6400-\mathrm{XT}$.

Leaf chemistry traits: On a different branch, all leaves were removed for bulk chemical analysis at different laboratories depending on the traits campaign.

Morphological and structural traits: Leaf area (hereafter referred to as Area) was determined by scanning the adaxial side of the leaf lamina on a flatbed scanner Canon LiDE220 ${ }^{\circledR}$ and analysing images with Matlab code available at https://github.com/bblonder/leafarea (see Neyret et al., 2016 for a full description of methodology). Trait values for compound leaves were analysed at the leaflet level. Specific leaf area (SLA) was determined by dividing leaf area by leaf dry mass. Leaf fresh mass (Fresh mass) was calculated as the fresh weight of the recently obtained leaves. Leaf dry mass (Dry mass) was determined after oven drying at $60{ }^{\circ} \mathrm{C}$ leaves for 72 hours or until constant weight. Leaf water content (LWC) was determined by subtracting the dry mass content from the fresh mass, multiplying by 100 and dividing by the fresh mass to obtain percentage water content. The thickness of leaf lamina (Thickness) was measured by taking four micrometre measurements halfway between the mid-vein and the edge of the leaf, avoiding major secondary veins. 
Table S1. Description of the European Space Agency Sentinel-2 satellite imagery used.

\begin{tabular}{|c|c|}
\hline Country & Sentinel-2 imagery selected \\
\hline \multirow[t]{4}{*}{ Malaysia } & S2A_MSIL1C_20170705T022551_N0205_R046_T50NML_20170705T024459.SAFE \\
\hline & S2A_MSIL1C_20160610T022552_N0202_R046_T50NNL_20160610T024605.SAFE \\
\hline & S2A_MSIL1C_20160918T022542_N0204_R046_T50NNL_20160918T024556.SAFE \\
\hline & S2A_MSIL1C_20180620T022551_N0206_R046_T50NNL_20180620T083834.SAFE \\
\hline \multirow[t]{6}{*}{ Australia } & S2A_MSIL1C_20160708T003032_N0204_R016_T55KCA_20160708T003035.SAFE \\
\hline & S2A_MSIL1C_20160926T003032_N0204_R016_T55KCA_20160926T003028.SAFE \\
\hline & S2A_MSIL1C_20160608T002712_N0202_R016_T55KCA_20160608T002733.SAFE \\
\hline & S2A_MSIL1C_20160502T003732_N0201_R059_T55KCA_20160502T003730.SAFE \\
\hline & S2A_MSIL1C_20161009T003952_N0204_R059_T55LCC_20161009T003951.SAFE \\
\hline & S2A_MSIL1C_20161009T003952_N0204_R059_T55KCB_20161009T003951.SAFE \\
\hline \multirow[t]{2}{*}{ Brazil -ST } & S2A_MSIL1C_20160701T140312_N0204_R067_T21MYS_20160701T140309.SAFE \\
\hline & S2A_MSIL1C_20150819T141046_N0204_R110_T21MYS_20150819T141044.SAFE \\
\hline Brazil -NX & S2A_MSIL1C_20160317T134032_N0201_R124_T22LCJ_20160317T134035.SAFE \\
\hline \multirow[t]{3}{*}{ Peru } & S2A_MSIL1C_20151020T144932_N0204_R139_T19LDF_20151020T144926.SAFE \\
\hline & S2A_MSIL1C_20160709T145732_N0204_R039_T19LBF_20160709T145757.SAFE \\
\hline & S2A_MSIL1C_20160917T150612_N0204_R039_T19LBG_20160917T150614.SAFE \\
\hline \multirow[t]{3}{*}{ Ghana } & S2A_MSIL1C_20151124T102342_N0204_R065_T30NXP_20151124T103016.SAFE \\
\hline & S2A_MSIL1C_20151224T102432_N0201_R065_T30NWL_20151224T103322.SAFE \\
\hline & S2A_MSIL1C_20151224T102432_N0201_R065_T30NXN_20151224T103322.SAFE \\
\hline \multirow[t]{3}{*}{ Gabon } & S2A_MSIL1C_20170402T093031_N0204_R136_T32NNF_20170402T093844.SAFE \\
\hline & S2A_MSIL1C_20170711T093031_N0205_R136_T32NNF_20170711T094456.SAFE \\
\hline & S2A_MSIL1C_20180124T092251_N0206_R093_T32MQE_20180125T172615.SAFE \\
\hline
\end{tabular}

All image tiles were downloaded from the European Space Agency (ESA) Sentinel-2 OpenHub https://scihub.copernicus.eu; Brazil -NX: Nova Xavantina; Brazil -ST: Santarem. 
Table S2. Comparison of trait distributions among tropical regions after running an analysis of variance and Tukey's HSD test to compare community weighted mean traits among locations. Nonsignificant differences are highlighted in grey.

\begin{tabular}{|c|c|c|c|c|c|}
\hline Trait & Locations & $\begin{array}{c}\text { Estimat } \\
\mathrm{e}\end{array}$ & Cl-Low & Cl-High & $\begin{array}{l}\text { Adj. } P \text { - } \\
\text { value }\end{array}$ \\
\hline \multirow{16}{*}{$\operatorname{Amax}(\mu \mathrm{mol} m-2 \mathrm{~s}-1)$} & Malaysia-Ghana & -0.7165 & -0.8100 & -0.6229 & $<0.001$ \\
\hline & Brazil NX-Ghana & 0.1793 & 0.0687 & 0.2899 & $<0.001$ \\
\hline & Brazil NX-Malaysia & 0.8957 & 0.7926 & 0.9988 & $<0.001$ \\
\hline & Peru-Ghana & -1.3638 & -1.4519 & -1.2758 & $<0.001$ \\
\hline & Peru-Malaysia & -0.6474 & -0.7259 & -0.5689 & $<0.001$ \\
\hline & Peru-Brazil NX & -1.5431 & -1.6413 & -1.4449 & $<0.001$ \\
\hline & Gabon-Australia & 2.5472 & 1.5720 & 3.5224 & $<0.001$ \\
\hline & Ghana-Australia & 3.7798 & 2.8664 & 4.6931 & $<0.001$ \\
\hline & Ghana-Gabon & 1.2325 & 0.3483 & 2.1168 & $<0.001$ \\
\hline & Malaysia-Australia & 7.5715 & 6.7262 & 8.4168 & $<0.001$ \\
\hline & Malaysia-Gabon & 5.0243 & 4.2106 & 5.8380 & $<0.001$ \\
\hline & Malaysia-Ghana & 3.7917 & 3.0533 & 4.5302 & $<0.001$ \\
\hline & Brazil NX-Australia & 2.0138 & 1.0376 & 2.9900 & $<0.001$ \\
\hline & Brazil NX-Gabon & -0.5334 & -1.4824 & 0.4156 & 0.6443 \\
\hline & Brazil NX-Ghana & -1.7660 & -2.6513 & -0.8806 & $<0.001$ \\
\hline & Brazil NX-Malaysia & -5.5577 & -6.3727 & -4.7428 & $<0.001$ \\
\hline \multirow[t]{15}{*}{ Area $(\mathrm{cm} 2)$} & Peru-Australia & 5.1447 & 4.3261 & 5.9633 & $<0.001$ \\
\hline & Peru-Gabon & 2.5975 & 1.8116 & 3.3834 & $<0.001$ \\
\hline & Peru-Ghana & 1.3649 & 0.6572 & 2.0727 & $<0.001$ \\
\hline & Peru-Malaysia & -2.4268 & -3.0442 & -1.8094 & $<0.001$ \\
\hline & Peru-Brazil NX & 3.1309 & 2.3437 & 3.9181 & $<0.001$ \\
\hline & Brazil ST-Australia & 3.4879 & 2.4057 & 4.5702 & $<0.001$ \\
\hline & Brazil ST-Gabon & 0.9407 & -0.1170 & 1.9984 & 0.1192 \\
\hline & Brazil ST-Ghana & -0.2918 & -1.2929 & 0.7092 & 0.9783 \\
\hline & Brazil ST-Malaysia & -4.0836 & -5.0229 & -3.1443 & $<0.001$ \\
\hline & Brazil ST-Brazil NX & 1.4741 & 0.4154 & 2.5328 & $<0.001$ \\
\hline & Brazil ST-Peru & -1.6568 & -2.5721 & -0.7414 & $<0.001$ \\
\hline & Ghana-Australia & 0.3792 & 0.2905 & 0.4678 & $<0.001$ \\
\hline & Malaysia-Australia & 0.0126 & -0.0706 & 0.0957 & 0.9940 \\
\hline & Malaysia-Ghana & -0.3666 & -0.4395 & -0.2937 & $<0.001$ \\
\hline & Brazil NX-Australia & 0.6046 & 0.5096 & 0.6996 & $<0.001$ \\
\hline \multirow{9}{*}{ Asat ( $\mu \mathrm{mol} m-2 \mathrm{~s}-1)$} & Brazil NX-Ghana & 0.2255 & 0.1393 & 0.3116 & $<0.001$ \\
\hline & Brazil NX-Malaysia & 0.5921 & 0.5115 & 0.6726 & $<0.001$ \\
\hline & Peru-Australia & -0.1918 & -0.2711 & -0.1125 & $<0.001$ \\
\hline & Peru-Ghana & -0.5710 & -0.6395 & -0.5024 & $<0.001$ \\
\hline & Peru-Malaysia & -0.2044 & -0.2656 & -0.1431 & $<0.001$ \\
\hline & Peru-Brazil NX & -0.7964 & -0.8729 & -0.7199 & $<0.001$ \\
\hline & Gabon-Australia & 0.3884 & 0.3566 & 0.4201 & $<0.001$ \\
\hline & Malaysia-Australia & -0.0400 & -0.0675 & -0.0124 & $<0.001$ \\
\hline & Malaysia-Gabon & -0.4283 & -0.4548 & -0.4018 & $<0.001$ \\
\hline \multirow{5}{*}{ C (\%) } & Peru-Australia & 0.2163 & 0.1896 & 0.2429 & $<0.001$ \\
\hline & Peru-Gabon & -0.1721 & -0.1977 & -0.1465 & $<0.001$ \\
\hline & Peru-Malaysia & 0.2562 & 0.2361 & 0.2763 & $<0.001$ \\
\hline & Brazil ST-Australia & 0.0765 & 0.0412 & 0.1118 & $<0.001$ \\
\hline & Brazil ST-Gabon & -0.3118 & -0.3464 & -0.2773 & $<0.001$ \\
\hline
\end{tabular}




\begin{tabular}{|c|c|c|c|c|c|}
\hline & Brazil ST-Malaysia & 0.1165 & 0.0858 & 0.1471 & $<0.001$ \\
\hline & Brazil ST-Peru & -0.1397 & -0.1696 & -0.1099 & $<0.001$ \\
\hline & Gabon-Australia & -0.1619 & -0.2118 & -0.1120 & $<0.001$ \\
\hline & Ghana-Australia & 0.2615 & 0.2149 & 0.3082 & $<0.001$ \\
\hline & Ghana-Gabon & 0.4234 & 0.3782 & 0.4687 & $<0.001$ \\
\hline & Malaysia-Australia & 0.0560 & 0.0128 & 0.0992 & 0.0026 \\
\hline & Malaysia-Gabon & 0.2179 & 0.1763 & 0.2596 & $<0.001$ \\
\hline & Malaysia-Ghana & -0.2055 & -0.2433 & -0.1678 & $<0.001$ \\
\hline & Brazil NX-Australia & -0.3203 & -0.3702 & -0.2704 & $<0.001$ \\
\hline & Brazil NX-Gabon & -0.1584 & -0.2069 & -0.1098 & $<0.001$ \\
\hline & Brazil NX-Ghana & -0.5818 & -0.6270 & -0.5366 & $<0.001$ \\
\hline & Brazil NX-Malaysia & -0.3763 & -0.4179 & -0.3347 & $<0.001$ \\
\hline $\mathrm{Ca}(\%)$ & Peru-Australia & -0.2092 & -0.2509 & -0.1674 & $<0.001$ \\
\hline & Peru-Gabon & -0.0473 & -0.0874 & -0.0071 & 0.0094 \\
\hline & Peru-Ghana & -0.4707 & -0.5068 & -0.4347 & $<0.001$ \\
\hline & Peru-Malaysia & -0.2652 & -0.2966 & -0.2337 & $<0.001$ \\
\hline & Peru-Brazil NX & 0.1111 & 0.0710 & 0.1512 & $<0.001$ \\
\hline & Brazil ST-Australia & -0.1213 & -0.1767 & -0.0660 & $<0.001$ \\
\hline & Brazil ST-Gabon & 0.0406 & -0.0135 & 0.0947 & 0.2894 \\
\hline & Brazil ST-Ghana & -0.3829 & -0.4340 & -0.3317 & $<0.001$ \\
\hline & Brazil ST-Malaysia & -0.1773 & -0.2254 & -0.1293 & $<0.001$ \\
\hline & Brazil ST-Brazil NX & 0.1989 & 0.1449 & 0.2530 & $<0.001$ \\
\hline & Brazil ST-Peru & 0.0878 & 0.0411 & 0.1345 & $<0.001$ \\
\hline & Malaysia-Gabon & 0.4365 & 0.3434 & 0.5297 & $<0.001$ \\
\hline & Brazil NX-Gabon & -0.0002 & -0.1088 & 0.1084 & 0.9999 \\
\hline & Brazil NX-Malaysia & -0.4367 & -0.5299 & -0.3435 & $<0.001$ \\
\hline & Peru-Gabon & 0.3140 & 0.2241 & 0.4040 & $<0.001$ \\
\hline $\operatorname{Dry}$ mass ( $\mathrm{g}$ ) & Peru-Malaysia & -0.1225 & -0.1932 & -0.0518 & $<0.001$ \\
\hline Dory mass (g) & Peru-Brazil NX & 0.3142 & 0.2242 & 0.4042 & $<0.001$ \\
\hline & Brazil ST-Gabon & 0.0027 & -0.1185 & 0.1239 & 0.9999 \\
\hline & Brazil ST-Malaysia & -0.4338 & -0.5415 & -0.3262 & $<0.001$ \\
\hline & Brazil ST-Brazil NX & 0.0029 & -0.1184 & 0.1241 & 0.9999 \\
\hline & Brazil ST-Peru & -0.3113 & -0.4162 & -0.2064 & $<0.001$ \\
\hline & Malaysia-Gabon & 0.7641 & 0.6500 & 0.8782 & $<0.001$ \\
\hline & Brazil NX-Gabon & -0.0187 & -0.1517 & 0.1144 & 0.9954 \\
\hline & Brazil NX-Malaysia & -0.7828 & -0.8970 & -0.6686 & $<0.001$ \\
\hline & Peru-Gabon & 0.1085 & -0.0017 & 0.2187 & 0.0559 \\
\hline Fresh mass $(\sigma)$ & Peru-Malaysia & -0.6556 & -0.7421 & -0.5691 & $<0.001$ \\
\hline 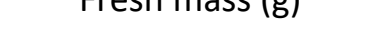 & Peru-Brazil NX & 0.1272 & 0.0169 & 0.2374 & 0.0143 \\
\hline & Brazil ST-Gabon & 0.0085 & -0.1400 & 0.1570 & 0.9999 \\
\hline & Brazil ST-Malaysia & -0.7556 & -0.8875 & -0.6237 & $<0.001$ \\
\hline & Brazil ST-Brazil NX & 0.0272 & -0.1214 & 0.1758 & 0.9874 \\
\hline & Brazil ST-Peru & -0.1000 & -0.2285 & 0.0285 & 0.2101 \\
\hline & Malaysia-Gabon & 0.3462 & 0.2193 & 0.4730 & $<0.001$ \\
\hline & Brazil NX-Gabon & -0.0373 & -0.1851 & 0.1105 & 0.9590 \\
\hline & Peru-Gabon & -0.1518 & -0.2804 & -0.0231 & 0.0113 \\
\hline Leaf water content (\%) & Brazil ST-Gabon & 0.0781 & -0.0872 & 0.2434 & 0.6976 \\
\hline & Brazil NX-Malaysia & -0.3835 & -0.5104 & -0.2565 & $<0.001$ \\
\hline & Peru-Malaysia & -0.4979 & -0.6019 & -0.3940 & $<0.001$ \\
\hline & Brazil ST-Malaysia & -0.2681 & -0.4150 & -0.1211 & $<0.001$ \\
\hline & Peru-Brazil NX & -0.1145 & -0.2432 & 0.0143 & 0.1083 \\
\hline
\end{tabular}




\begin{tabular}{|c|c|c|c|c|c|}
\hline & Brazil ST-Brazil NX & 0.1154 & -0.0500 & 0.2808 & 0.3151 \\
\hline & Brazil ST-Peru & 0.2299 & 0.0814 & 0.3784 & $<0.001$ \\
\hline & Gabon-Australia & -0.0136 & -0.0436 & 0.0164 & 0.8364 \\
\hline & Ghana-Australia & 0.1518 & 0.1238 & 0.1799 & $<0.001$ \\
\hline & Ghana-Gabon & 0.1654 & 0.1382 & 0.1926 & $<0.001$ \\
\hline & Malaysia-Australia & 0.1738 & 0.1478 & 0.1998 & $<0.001$ \\
\hline & Malaysia-Gabon & 0.1874 & 0.1623 & 0.2124 & $<0.001$ \\
\hline & Malaysia-Ghana & 0.0220 & -0.0007 & 0.0447 & 0.0649 \\
\hline & Brazil NX-Australia & -0.2413 & -0.2713 & -0.2114 & $<0.001$ \\
\hline & Brazil NX-Gabon & -0.2278 & -0.2570 & -0.1986 & $<0.001$ \\
\hline & Brazil NX-Ghana & -0.3932 & -0.4203 & -0.3660 & $<0.001$ \\
\hline & Brazil NX-Malaysia & -0.4151 & -0.4402 & -0.3901 & $<0.001$ \\
\hline K (\%) & Peru-Australia & -0.1111 & -0.1362 & -0.0860 & $<0.001$ \\
\hline & Peru-Gabon & -0.0976 & -0.1217 & -0.0734 & $<0.001$ \\
\hline & Peru-Ghana & -0.2629 & -0.2846 & -0.2413 & $<0.001$ \\
\hline & Peru-Malaysia & -0.2849 & -0.3038 & -0.2660 & $<0.001$ \\
\hline & Peru-Brazil NX & 0.1302 & 0.1061 & 0.1543 & $<0.001$ \\
\hline & Brazil ST-Australia & -0.2181 & -0.2513 & -0.1848 & $<0.001$ \\
\hline & Brazil ST-Gabon & -0.2045 & -0.2370 & -0.1720 & $<0.001$ \\
\hline & Brazil ST-Ghana & -0.3699 & -0.4006 & -0.3391 & $<0.001$ \\
\hline & Brazil ST-Malaysia & -0.3918 & -0.4207 & -0.3630 & $<0.001$ \\
\hline & Brazil ST-Brazil NX & 0.0233 & -0.0092 & 0.0558 & 0.3453 \\
\hline & Brazil ST-Peru & -0.1069 & -0.1350 & -0.0789 & $<0.001$ \\
\hline & Gabon-Australia & -0.0373 & -0.0584 & -0.0162 & $<0.001$ \\
\hline & Ghana-Australia & 0.0533 & 0.0335 & 0.0730 & $<0.001$ \\
\hline & Ghana-Gabon & 0.0906 & 0.0714 & 0.1097 & $<0.001$ \\
\hline & Malaysia-Australia & 0.0030 & -0.0153 & 0.0213 & 0.9991 \\
\hline & Malaysia-Gabon & 0.0403 & 0.0226 & 0.0579 & $<0.001$ \\
\hline & Malaysia-Ghana & -0.0503 & -0.0663 & -0.0343 & $<0.001$ \\
\hline & Brazil NX-Australia & -0.1297 & -0.1508 & -0.1086 & $<0.001$ \\
\hline & Brazil NX-Gabon & -0.0924 & -0.1129 & -0.0718 & $<0.001$ \\
\hline & Brazil NX-Ghana & -0.1830 & -0.2021 & -0.1638 & $<0.001$ \\
\hline & Brazil NX-Malaysia & -0.1327 & -0.1503 & -0.1150 & $<0.001$ \\
\hline Mg (\%) & Peru-Australia & -0.0184 & -0.0361 & -0.0008 & 0.0345 \\
\hline & Peru-Gabon & 0.0189 & 0.0019 & 0.0359 & 0.0181 \\
\hline & Peru-Ghana & -0.0717 & -0.0870 & -0.0564 & $<0.001$ \\
\hline & Peru-Malaysia & -0.0214 & -0.0347 & -0.0081 & $<0.001$ \\
\hline & Peru-Brazil NX & 0.1113 & 0.0943 & 0.1282 & $<0.001$ \\
\hline & Brazil ST-Australia & -0.0938 & -0.1172 & -0.0704 & $<0.001$ \\
\hline & Brazil ST-Gabon & -0.0565 & -0.0794 & -0.0336 & $<0.001$ \\
\hline & Brazil ST-Ghana & -0.1471 & -0.1687 & -0.1255 & $<0.001$ \\
\hline & Brazil ST-Malaysia & -0.0968 & -0.1171 & -0.0765 & $<0.001$ \\
\hline & Brazil ST-Brazil NX & 0.0359 & 0.0130 & 0.0588 & $<0.001$ \\
\hline & Brazil ST-Peru & -0.0754 & -0.0952 & -0.0556 & $<0.001$ \\
\hline & Gabon-Australia & 0.2718 & 0.2422 & 0.3015 & $<0.001$ \\
\hline & Ghana-Australia & 0.3936 & 0.3658 & 0.4214 & $<0.001$ \\
\hline & Ghana-Gabon & 0.1218 & 0.0949 & 0.1487 & $<0.001$ \\
\hline $\mathrm{N}(\%)$ & Malaysia-Australia & 0.2296 & 0.2039 & 0.2553 & $<0.001$ \\
\hline & Malaysia-Gabon & -0.0422 & -0.0670 & -0.0174 & $<0.001$ \\
\hline & Malaysia-Ghana & -0.1640 & -0.1864 & -0.1415 & $<0.001$ \\
\hline & Brazil NX-Australia & 0.3179 & 0.2882 & 0.3476 & $<0.001$ \\
\hline
\end{tabular}




\begin{tabular}{|c|c|c|c|c|c|}
\hline & Brazil NX-Gabon & 0.0461 & 0.0172 & 0.0749 & $<0.001$ \\
\hline & Brazil NX-Ghana & -0.0757 & -0.1026 & -0.0488 & $<0.001$ \\
\hline & Brazil NX-Malaysia & 0.0883 & 0.0635 & 0.1131 & $<0.001$ \\
\hline & Peru-Australia & 0.2982 & 0.2733 & 0.3231 & $<0.001$ \\
\hline & Peru-Gabon & 0.0264 & 0.0024 & 0.0503 & 0.0199 \\
\hline & Peru-Ghana & -0.0954 & -0.1169 & -0.0739 & $<0.001$ \\
\hline & Peru-Malaysia & 0.0686 & 0.0498 & 0.0874 & $<0.001$ \\
\hline & Peru-Brazil NX & -0.0197 & -0.0436 & 0.0042 & 0.1861 \\
\hline & Brazil ST-Australia & 0.3348 & 0.3018 & 0.3677 & $<0.001$ \\
\hline & Brazil ST-Gabon & 0.0629 & 0.0307 & 0.0952 & $<0.001$ \\
\hline & Brazil ST-Ghana & -0.0588 & -0.0893 & -0.0283 & $<0.001$ \\
\hline & Brazil ST-Malaysia & 0.1052 & 0.0765 & 0.1338 & $<0.001$ \\
\hline & Brazil ST-Brazil NX & 0.0169 & -0.0154 & 0.0491 & 0.7184 \\
\hline & Brazil ST-Peru & 0.0366 & 0.0087 & 0.0645 & 0.0022 \\
\hline & Gabon-Australia & 0.0026 & -0.0070 & 0.0123 & 0.9846 \\
\hline & Ghana-Australia & 0.0707 & 0.0617 & 0.0797 & $<0.001$ \\
\hline & Ghana-Gabon & 0.0681 & 0.0593 & 0.0768 & $<0.001$ \\
\hline & Malaysia-Australia & 0.0692 & 0.0609 & 0.0776 & $<0.001$ \\
\hline & Malaysia-Gabon & 0.0666 & 0.0586 & 0.0747 & $<0.001$ \\
\hline & Malaysia-Ghana & -0.0015 & -0.0087 & 0.0058 & 0.9972 \\
\hline & Brazil NX-Australia & 0.0597 & 0.0501 & 0.0694 & $<0.001$ \\
\hline & Brazil NX-Gabon & 0.0571 & 0.0477 & 0.0665 & $<0.001$ \\
\hline & Brazil NX-Ghana & -0.0110 & -0.0197 & -0.0022 & 0.0041 \\
\hline & Brazil NX-Malaysia & -0.0095 & -0.0176 & -0.0015 & 0.0090 \\
\hline $\mathrm{P}(\%)$ & Peru-Australia & 0.0647 & 0.0567 & 0.0728 & $<0.001$ \\
\hline & Peru-Gabon & 0.0621 & 0.0543 & 0.0699 & $<0.001$ \\
\hline & Peru-Ghana & -0.0060 & -0.0129 & 0.0010 & 0.1537 \\
\hline & Peru-Malaysia & -0.0045 & -0.0106 & 0.0016 & 0.3062 \\
\hline & Peru-Brazil NX & 0.0050 & -0.0028 & 0.0128 & 0.4789 \\
\hline & Brazil ST-Australia & 0.0219 & 0.0112 & 0.0326 & $<0.001$ \\
\hline & Brazil ST-Gabon & 0.0193 & 0.0088 & 0.0298 & $<0.001$ \\
\hline & Brazil ST-Ghana & -0.0488 & -0.0586 & -0.0389 & $<0.001$ \\
\hline & Brazil ST-Malaysia & -0.0473 & -0.0566 & -0.0380 & $<0.001$ \\
\hline & Brazil ST-Brazil NX & -0.0378 & -0.0483 & -0.0273 & $<0.001$ \\
\hline & Brazil ST-Peru & -0.0428 & -0.0518 & -0.0338 & $<0.001$ \\
\hline & Gabon-Australia & 0.0118 & 0.0086 & 0.0150 & $<0.001$ \\
\hline & Ghana-Australia & 0.0114 & 0.0084 & 0.0144 & $<0.001$ \\
\hline & Ghana-Gabon & -0.0004 & -0.0033 & 0.0025 & 0.9997 \\
\hline & Malaysia-Australia & 0.0142 & 0.0114 & 0.0170 & $<0.001$ \\
\hline & Malaysia-Gabon & 0.0023 & -0.0003 & 0.0050 & 0.1295 \\
\hline & Malaysia-Ghana & 0.0027 & 0.0003 & 0.0052 & 0.0150 \\
\hline & Brazil NX-Australia & 0.0021 & -0.0011 & 0.0053 & 0.4573 \\
\hline$\left(m^{2} g-1\right)$ & Brazil NX-Gabon & -0.0097 & -0.0128 & -0.0066 & $<0.001$ \\
\hline JLA (IIIL $5-1)$ & Brazil NX-Ghana & -0.0093 & -0.0122 & -0.0064 & $<0.001$ \\
\hline & Brazil NX-Malaysia & -0.0121 & -0.0148 & -0.0094 & $<0.001$ \\
\hline & Peru-Australia & 0.0071 & 0.0044 & 0.0098 & $<0.001$ \\
\hline & Peru-Gabon & -0.0047 & -0.0073 & -0.0022 & $<0.001$ \\
\hline & Peru-Ghana & -0.0043 & -0.0067 & -0.0020 & $<0.001$ \\
\hline & Peru-Malaysia & -0.0071 & -0.0091 & -0.0051 & $<0.001$ \\
\hline & Peru-Brazil NX & 0.0050 & 0.0024 & 0.0076 & $<0.001$ \\
\hline & Brazil ST-Australia & 0.0175 & 0.0139 & 0.0210 & $<0.001$ \\
\hline
\end{tabular}




\begin{tabular}{llrrrr} 
& Brazil ST-Gabon & 0.0057 & 0.0022 & 0.0091 & $<0.001$ \\
Brazil ST-Ghana & 0.0061 & 0.0028 & 0.0093 & $<0.001$ \\
Brazil ST-Malaysia & 0.0033 & 0.0002 & 0.0064 & 0.0262 \\
Brazil ST-Brazil NX & 0.0154 & 0.0119 & 0.0189 & $<0.001$ \\
Brazil ST-Peru & 0.0104 & 0.0074 & 0.0134 & $<0.001$ \\
\hline Gabon-Australia & 0.0151 & -0.0018 & 0.0321 & 0.1165 \\
Ghana-Australia & -0.0037 & -0.0196 & 0.0122 & 0.9932 \\
\hline Ghana-Gabon & -0.0188 & -0.0342 & -0.0035 & 0.0056 \\
Malaysia-Australia & -0.0208 & -0.0355 & -0.0061 & $<0.001$ \\
Malaysia-Gabon & -0.0360 & -0.0501 & -0.0218 & $<0.001$ \\
Malaysia-Ghana & -0.0171 & -0.0299 & -0.0043 & 0.0016 \\
Brazil NX-Australia & 0.0191 & 0.0022 & 0.0361 & 0.0155 \\
\hline Brazil NX-Gabon & 0.0040 & -0.0125 & 0.0205 & 0.9918 \\
\hline Brazil NX-Ghana & 0.0228 & 0.0075 & 0.0382 & $<0.001$ \\
Brazil NX-Malaysia & 0.0400 & 0.0258 & 0.0541 & $<0.001$ \\
Peru-Australia & 0.0385 & 0.0243 & 0.0528 & $<0.001$ \\
Peru-Gabon & 0.0234 & 0.0098 & 0.0371 & $<0.001$ \\
Peru-Ghana & 0.0423 & 0.0300 & 0.0545 & $<0.001$ \\
Peru-Malaysia & 0.0594 & 0.0486 & 0.0701 & $<0.001$ \\
Peru-Brazil NX & 0.0194 & 0.0058 & 0.0331 & $<0.001$ \\
\hline Brazil ST-Australia & -0.0166 & -0.0355 & 0.0022 & 0.1232 \\
\hline Brazil ST-Gabon & -0.0318 & -0.0502 & -0.0134 & $<0.001$ \\
\hline Brazil ST-Ghana & -0.0129 & -0.0303 & 0.0045 & 0.2996 \\
Brazil ST-Malaysia & 0.0042 & -0.0121 & 0.0205 & 0.9888 \\
\hline Brazil ST-Brazil NX & -0.0358 & -0.0542 & -0.0174 & $<0.001$ \\
Brazil ST-Peru & -0.0552 & -0.0711 & -0.0393 & $<0.001$ \\
\hline
\end{tabular}

Brazil -NX: Nova Xavantina; Brazil -ST: Santarem. 
Table S3. Statistical results for the global trait distribution models constructed with the smaller dataset where the 25th and

50th percentile of smallest trees were removed. The correlation to the results obtained with the full dataset (without removing the smallest trees) is shown.

\begin{tabular}{|c|c|c|c|c|c|c|c|}
\hline Type & Dataset & Trait & MAE & MSE & RMSE & $\mathbf{R}^{2}$ & $\begin{array}{l}\text { Correlation to } \\
\text { full dataset (R2) }\end{array}$ \\
\hline \multirow{8}{*}{$\begin{array}{l}\text { Morphological and } \\
\text { water content }\end{array}$} & \multirow{15}{*}{$\begin{array}{c}25 \% \text { smallest trees } \\
\text { out }\end{array}$} & & & 1360.31106 & 36.88 & & \multirow{15}{*}{0.95} \\
\hline & & Area $(\mathrm{cm} 2)$ & 26.71 & 3 & 2 & 0.45 & \\
\hline & & Dry mass (g) & 0.377 & 0.256267 & 0.506 & 0.27 & \\
\hline & & Fresh mass (g) & 0.857 & 1.34205 & 1.158 & 0.28 & \\
\hline & & $\mathrm{SLA}(\mathrm{m} 2 \mathrm{~g}-1)$ & 0.001 & 3.00E-06 & 0.002 & 0.39 & \\
\hline & & Thickness (mm) & 0.036 & 0.002358 & 0.049 & 0.51 & \\
\hline & & Leaf water content (\%) & 4.146 & 30.686676 & 5.54 & 0.32 & \\
\hline & & $C(\%)$ & 1.364 & 3.358402 & 1.833 & 0.61 & \\
\hline \multirow{5}{*}{ Chemical } & & $\mathrm{Ca}(\%)$ & 0.153 & 0.045916 & 0.214 & 0.59 & \\
\hline & & K (\%) & 0.13 & 0.030804 & 0.176 & 0.64 & \\
\hline & & Mg (\%) & 0.056 & 0.005731 & 0.076 & 0.48 & \\
\hline & & $\mathrm{N}(\%)$ & 0.238 & 0.09876 & 0.314 & 0.56 & \\
\hline & & $\mathrm{P}(\%)$ & 0.016 & 4.49E-04 & 0.021 & 0.56 & \\
\hline \multirow{2}{*}{ Photosynthetic } & & Amax $(\mu \mathrm{mol} m-2 \mathrm{~s}-1)$ & 3.11 & 17.122803 & 4.138 & 0.64 & \\
\hline & & Asat ( $\mu \mathrm{mol} m-2 \mathrm{~s}-1)$ & 1.379 & 3.293167 & 1.815 & 0.58 & \\
\hline \multirow{8}{*}{$\begin{array}{l}\text { Morphological and } \\
\text { water content }\end{array}$} & \multirow{12}{*}{$\begin{array}{c}50 \% \text { smallest trees } \\
\text { out }\end{array}$} & & 29.09 & 1601.00479 & 40.01 & & \multirow{12}{*}{0.97} \\
\hline & & Area $(\mathrm{cm} 2)$ & 3 & 8 & 3 & 0.37 & \\
\hline & & Dry mass (g) & 0.387 & 0.263549 & 0.513 & 0.27 & \\
\hline & & Fresh mass (g) & 0.838 & 1.204538 & 1.098 & 0.27 & \\
\hline & & SLA (m2 g-1) & 0.001 & 3.00E-06 & 0.002 & 0.4 & \\
\hline & & Thickness (mm) & 0.038 & 0.00252 & 0.05 & 0.52 & \\
\hline & & Leaf water content (\%) & 4.144 & 30.736321 & 5.544 & 0.32 & \\
\hline & & $C(\%)$ & 1.374 & 3.559623 & 1.887 & 0.58 & \\
\hline \multirow{4}{*}{ Chemical } & & $\mathrm{Ca}(\%)$ & 0.162 & 0.051693 & 0.227 & 0.59 & \\
\hline & & $\mathrm{K}(\%)$ & 0.145 & 0.038401 & 0.196 & 0.57 & \\
\hline & & $\mathrm{Mg}(\%)$ & 0.06 & 0.00633 & 0.08 & 0.42 & \\
\hline & & $\mathrm{N}(\%)$ & 0.255 & 0.110082 & 0.332 & 0.52 & \\
\hline
\end{tabular}




\begin{tabular}{llrrrr} 
& $\mathrm{P}(\%)$ & 0.016 & $4.53 \mathrm{E}-04$ & 0.021 & 0.55 \\
\cline { 2 - 5 } Photosynthetic & Amax $(\mu \mathrm{mol} \mathrm{m}-2 \mathrm{~s}-1)$ & 3.437 & 20.261913 & 4.501 & 0.6 \\
& Asat $(\mu \mathrm{mol} \mathrm{m}-2 \mathrm{~s}-1)$ & 1.452 & 3.734509 & 1.932 & 0.54
\end{tabular}

MAE: Mean Absolute Error; MSE: Mean Square Error; RMSE: Root mean square error. 
Table S4. Statistical results for the models fitted per region for each functional trait constructed with the smaller dataset where the 25th and 50th percentile of smallest trees was removed. The correlation to the results obtained with the full dataset (without removing the smallest trees) per trait and the overall correlation over all traits across regions is shown.

\begin{tabular}{|c|c|c|c|c|c|c|c|c|}
\hline Locations & Dataset & Trait & MAE & MSE & RMSE & $\mathbf{R}^{2}$ & $\begin{array}{l}\text { Correlation to } \\
\text { full dataset }\end{array}$ & $\begin{array}{c}\text { Overall } \\
\text { correlation to full } \\
\text { dataset }\end{array}$ \\
\hline \multirow{14}{*}{ Malaysia } & $\begin{array}{c}25 \% \text { smallest trees } \\
\text { out }\end{array}$ & $\operatorname{Amax}(\mu \mathrm{mol} m-2 \mathrm{~s}-1)$ & 4.4075 & 30.8047 & 5.5502 & 0.42 & \multirow{14}{*}{0.86} & \multirow{24}{*}{0.81} \\
\hline & & Area $(\mathrm{cm} 2)$ & 149.4096 & 71974.0935 & 268.2799 & 0.07 & & \\
\hline & & Asat $(\mu \mathrm{mol} m-2 \mathrm{~s}-1)$ & 1.86 & 5.6441 & 2.3757 & 0.44 & & \\
\hline & & C (\%) & 1.5683 & 4.8068 & 2.1924 & 0.27 & & \\
\hline & & $\mathrm{Ca}(\%)$ & 0.2704 & 0.3459 & 0.5881 & 0.31 & & \\
\hline & & Dry mass (g) & 1.7823 & 11.9282 & 3.4537 & 0.04 & & \\
\hline & & Fresh mass (g) & 4.3969 & 72.2848 & 8.502 & 0.04 & & \\
\hline & & Leaf water content (\%) & 3.8173 & 26.2822 & 5.1266 & 0.19 & & \\
\hline & & $\mathrm{K}(\%)$ & 0.2888 & 0.1736 & 0.4166 & 0.27 & & \\
\hline & & $\mathrm{Mg}(\%)$ & 0.0968 & 0.0176 & 0.1326 & 0.22 & & \\
\hline & & $\mathrm{N}(\%)$ & 0.2589 & 0.1483 & 0.3851 & 0.19 & & \\
\hline & & $\mathrm{P}(\%)$ & 0.0255 & 0.0024 & 0.0487 & 0.16 & & \\
\hline & & $\operatorname{SLA}(m 2 g-1)$ & 0.0016 & 0 & 0.0024 & 0.11 & & \\
\hline & & Thickness (mm) & 0.0543 & 0.0094 & 0.097 & 0.26 & & \\
\hline \multirow{10}{*}{ Brazil -NX } & & $\operatorname{Amax}(\mu \mathrm{mol} m-2 \mathrm{~s}-1)$ & 2.7177 & 11.8535 & 3.4429 & 0.42 & \multirow{10}{*}{0.95} & \\
\hline & & Area $(\mathrm{cm} 2)$ & 16.4847 & 642.7476 & 25.3525 & 0.09 & & \\
\hline & & Asat $(\mu \mathrm{mol} m-2 \mathrm{~s}-1)$ & 1.5203 & 3.5129 & 1.8743 & 0.59 & & \\
\hline & & $\mathrm{Ca}(\%)$ & 0.0701 & 0.0092 & 0.0961 & 0.43 & & \\
\hline & & Dry mass (g) & 0.2416 & 0.1682 & 0.4101 & 0.29 & & \\
\hline & & Fresh mass (g) & 0.6086 & 1.2114 & 1.1006 & 0.24 & & \\
\hline & & Leaf water content (\%) & 3.0527 & 18.5636 & 4.3085 & 0.06 & & \\
\hline & & $\mathrm{K}(\%)$ & 0.0743 & 0.0112 & 0.1058 & 0.14 & & \\
\hline & & $\mathrm{Mg}(\%)$ & 0.0207 & $7.00 \mathrm{E}-04$ & 0.0267 & 0.61 & & \\
\hline & & $\mathrm{N}(\%)$ & 0.1737 & 0.0506 & 0.2249 & 0.51 & & \\
\hline
\end{tabular}




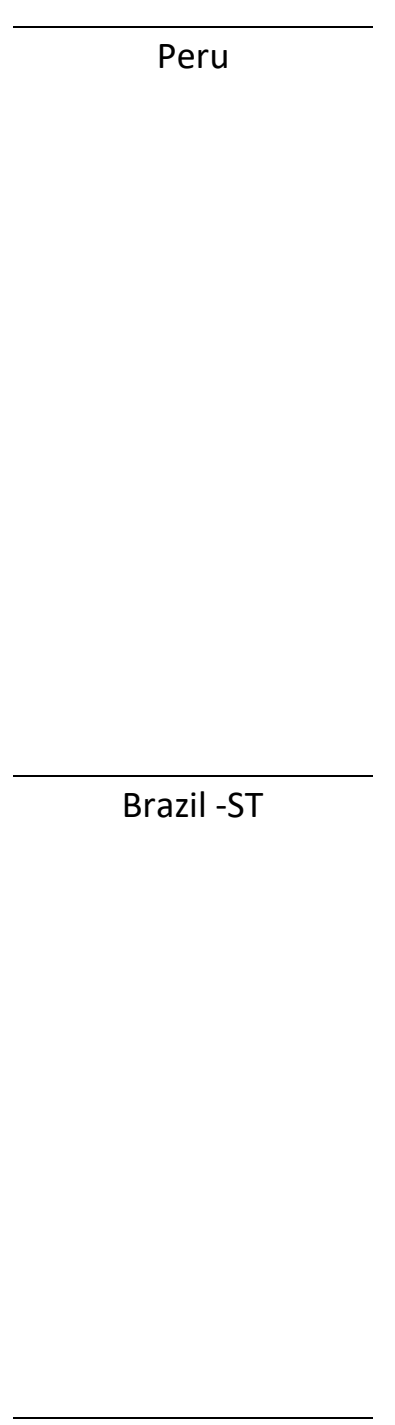

Ghana

\begin{tabular}{|c|c|c|c|c|c|}
\hline & & & & & \multirow{18}{*}{0.92} \\
\hline $\mathrm{P}(\%)$ & 0.0146 & $3.00 \mathrm{E}-04$ & 0.0183 & 0.64 & \\
\hline $\operatorname{SLA}(m 2 g-1)$ & 8.00E-04 & 0 & 0.0011 & 0.55 & \\
\hline Thickness (mm) & 0.0242 & 0.0011 & 0.0329 & 0.65 & \\
\hline $\operatorname{Amax}(\mu \mathrm{mol} m-2 \mathrm{~s}-1)$ & 2.698 & 13.1243 & 3.6228 & 0.38 & \\
\hline Area $(\mathrm{cm} 2)$ & 93.2267 & 30998.2922 & 176.0633 & 0.26 & \\
\hline Asat ( $\mu \mathrm{mol} \mathrm{m}-2 \mathrm{~s}-1)$ & 1.483 & 3.855 & 1.9634 & 0.39 & \\
\hline $\mathrm{C}(\%)$ & 1.4817 & 4.104 & 2.0258 & 0.29 & \\
\hline $\mathrm{Ca}(\%)$ & 0.1357 & 0.0365 & 0.191 & 0.68 & \\
\hline Dry mass (g) & 1.2982 & 5.8398 & 2.4166 & 0.14 & \\
\hline Fresh mass (g) & 1.068 & 2.3976 & 1.5484 & 0.22 & \\
\hline Leaf water content (\%) & 10.1376 & 219.9969 & 14.8323 & 0.33 & \\
\hline $\mathrm{K}(\%)$ & 0.1059 & 0.0197 & 0.1403 & 0.26 & \\
\hline $\mathrm{Mg}(\%)$ & 0.0595 & 0.0075 & 0.0865 & 0.48 & \\
\hline $\mathrm{N}(\%)$ & 0.2524 & 0.1082 & 0.3289 & 0.47 & \\
\hline $\mathrm{P}(\%)$ & 0.0216 & $9.00 \mathrm{E}-04$ & 0.0303 & 0.45 & \\
\hline SLA (m2 g-1) & 0.0031 & 0 & 0.0059 & 0.38 & \\
\hline Thickness (mm) & 0.0559 & 0.0065 & 0.0805 & 0.73 & \\
\hline Area $(\mathrm{cm} 2)$ & 47.4017 & 5042.7689 & 71.0125 & 0.01 & \\
\hline $\mathrm{C}(\%)$ & 1.3916 & 3.1239 & 1.7675 & 0.11 & \\
\hline $\mathrm{Ca}(\%)$ & 0.2632 & 0.1094 & 0.3308 & 0.03 & \\
\hline Dry mass (g) & 0.4504 & 0.4184 & 0.6468 & 0.01 & \\
\hline Fresh mass (g) & 1.0677 & 2.6544 & 1.6292 & 0.31 & \\
\hline Leaf water content (\%) & 4.0807 & 32.3078 & 5.684 & 0.09 & 0.72 \\
\hline $\mathrm{K}(\%)$ & 0.1252 & 0.0288 & 0.1697 & 0.33 & \\
\hline $\mathrm{Mg}(\%)$ & 0.057 & 0.0053 & 0.0728 & 0.25 & \\
\hline $\mathrm{N}(\%)$ & 0.382 & 0.2621 & 0.512 & 0.25 & \\
\hline$P(\%)$ & 0.0245 & 0.0011 & 0.0338 & 0.34 & \\
\hline $\mathrm{SLA}(\mathrm{m} 2 \mathrm{~g}-1)$ & 0.0019 & 0 & 0.0025 & 0.21 & \\
\hline Thickness (mm) & 0.0503 & 0.0053 & 0.0727 & 0.29 & \\
\hline $\operatorname{Amax}(\mu \mathrm{mol} m-2 \mathrm{~s}-1)$ & 2.2485 & 9.538 & 3.0884 & 0.29 & \\
\hline Area $(\mathrm{cm} 2)$ & 36.2236 & 2725.1935 & 52.2034 & 0.37 & 0.42 \\
\hline Asat ( $\mu \mathrm{mol} \mathrm{m}-2 \mathrm{~s}-1)$ & 1.3194 & 3.6126 & 1.9007 & 0.11 & \\
\hline
\end{tabular}




\begin{tabular}{|c|c|c|c|c|c|c|c|c|}
\hline & & $\mathrm{Ca}(\%)$ & 0.3773 & 0.3294 & 0.574 & 0.57 & & \\
\hline & & K (\%) & 0.2965 & 0.1509 & 0.3884 & 0.3 & & \\
\hline & & $\mathrm{Mg}(\%)$ & 0.0947 & 0.0137 & 0.1172 & 0.29 & & \\
\hline & & $\mathrm{N}(\%)$ & 0.3622 & 0.6545 & 0.809 & 0.22 & & \\
\hline & & $\mathrm{P}(\%)$ & 0.0182 & $7.00 \mathrm{E}-04$ & 0.0255 & 0.42 & & \\
\hline & & SLA (m2 g-1) & 0.0014 & 0 & 0.0018 & 0.24 & & \\
\hline & & Thickness (mm) & 0.0308 & 0.0017 & 0.0406 & 0.09 & & \\
\hline \multirow{10}{*}{ Australia } & & Area $(\mathrm{cm} 2)$ & 8.4681 & 202.7337 & 14.2385 & 0.29 & \multirow{10}{*}{0.68} & \\
\hline & & Asat $(\mu \mathrm{mol} m-2 \mathrm{~s}-1)$ & 0.7602 & 1.026 & 1.0129 & 0.08 & & \\
\hline & & C (\%) & 1.0098 & 1.8311 & 1.3532 & 0.38 & & \\
\hline & & Ca (\%) & 0.2379 & 0.1211 & 0.348 & 0.16 & & \\
\hline & & K (\%) & 0.1366 & 0.0318 & 0.1783 & 0.13 & & \\
\hline & & $\mathrm{Mg}(\%)$ & 0.0703 & 0.0098 & 0.0991 & 0.06 & & \\
\hline & & $\mathrm{N}(\%)$ & 0.1511 & 0.0418 & 0.2045 & 0.1 & & \\
\hline & & $\mathrm{P}(\%)$ & 0.0096 & $2.00 \mathrm{E}-04$ & 0.0136 & 0.17 & & \\
\hline & & SLA (m2 g-1) & 0.0011 & 0 & 0.0014 & 0.19 & & \\
\hline & & Thickness (mm) & 0.038 & 0.0024 & 0.0492 & 0.36 & & \\
\hline \multirow{12}{*}{ Gabon } & & Area $(\mathrm{cm} 2)$ & 26.2327 & 1598.5937 & 39.9824 & 0.47 & \multirow{12}{*}{0.66} & \\
\hline & & C (\%) & 1.3224 & 3.2113 & 1.792 & 0.18 & & \\
\hline & & Сa (\%) & 0.1744 & 0.0548 & 0.2342 & 0.36 & & \\
\hline & & Dry mass (g) & 0.4585 & 0.7355 & 0.8576 & 0.4 & & \\
\hline & & Fresh mass (g) & 0.9372 & 5.5005 & 2.3453 & 0.27 & & \\
\hline & & Leaf water content (\%) & 4.6734 & 47.67 & 6.9043 & 0.48 & & \\
\hline & & K (\%) & 0.1804 & 0.0723 & 0.2688 & 0.26 & & \\
\hline & & Mg (\%) & 0.0803 & 0.0113 & 0.1061 & 0.41 & & \\
\hline & & $\mathrm{N}(\%)$ & 0.3009 & 0.1669 & 0.4085 & 0.51 & & \\
\hline & & $\mathrm{P}(\%)$ & 0.0082 & $1.00 \mathrm{E}-04$ & 0.0113 & 0.59 & & \\
\hline & & SLA (m2 g-1) & 0.0019 & 0 & 0.0028 & 0.22 & & \\
\hline & & Thickness (mm) & 0.05 & 0.0052 & 0.0723 & 0.18 & & \\
\hline \multirow{3}{*}{\multicolumn{2}{|c|}{$\begin{array}{r}\text { D\% smalles } \\
\text { out }\end{array}$}} & Amax $(\mu \mathrm{mol} m-2 \mathrm{~s}-1)$ & 4.2786 & 29.9285 & 5.4707 & 0.44 & \multirow{3}{*}{0.90} & \multirow{3}{*}{0.80} \\
\hline & & Area $(\mathrm{cm} 2)$ & 136.9901 & 57104.3867 & 238.9652 & 0.06 & & \\
\hline & & Asat $(\mu \mathrm{mol} m-2 \mathrm{~s}-1)$ & 2.0399 & 7.1341 & 2.671 & 0.33 & & \\
\hline
\end{tabular}




\begin{tabular}{|c|c|c|c|c|c|c|}
\hline & $C(\%)$ & 1.6566 & 5.1133 & 2.2613 & 0.31 & \\
\hline & $\mathrm{Ca}(\%)$ & 0.3117 & 0.4211 & 0.649 & 0.33 & \\
\hline & Dry mass (g) & 1.3783 & 4.2579 & 2.0635 & 0.11 & \\
\hline & Fresh mass (g) & 3.7924 & 50.5755 & 7.1116 & 0.04 & \\
\hline & Leaf water content (\%) & 3.5339 & 24.5958 & 4.9594 & 0.18 & \\
\hline & $\mathrm{K}(\%)$ & 0.3152 & 0.2012 & 0.4485 & 0.2 & \\
\hline & Mg (\%) & 0.0926 & 0.0186 & 0.1363 & 0.38 & \\
\hline & $\mathrm{N}(\%)$ & 0.2807 & 0.155 & 0.3938 & 0.14 & \\
\hline & $\mathrm{P}(\%)$ & 0.0218 & 0.0016 & 0.0399 & 0.3 & \\
\hline & $\mathrm{SLA}(\mathrm{m} 2 \mathrm{~g}-1)$ & 0.0017 & 0 & 0.0024 & 0.11 & \\
\hline & Thickness (mm) & 0.0586 & 0.0118 & 0.1086 & 0.23 & \\
\hline \multirow{13}{*}{ Brazil -NX } & $\operatorname{Amax}(\mu \mathrm{mol} m-2 \mathrm{~s}-1)$ & 3.2298 & 19.8841 & 4.4592 & 0.36 & \multirow{13}{*}{0.92} \\
\hline & Area $(\mathrm{cm} 2)$ & 19.3015 & 957.0967 & 30.937 & 0.13 & \\
\hline & Asat $(\mu \mathrm{mol} m-2 \mathrm{~s}-1)$ & 1.6448 & 4.5215 & 2.1264 & 0.54 & \\
\hline & $\mathrm{Ca}(\%)$ & 0.0718 & 0.014 & 0.1185 & 0.38 & \\
\hline & Dry mass (g) & 0.312 & 0.2804 & 0.5295 & 0.15 & \\
\hline & Fresh mass (g) & 0.6595 & 1.0529 & 1.0261 & 0.21 & \\
\hline & Leaf water content (\%) & 3.8178 & 24.3504 & 4.9346 & 0.12 & \\
\hline & $\mathrm{K}(\%)$ & 0.0755 & 0.01 & 0.1001 & 0.03 & \\
\hline & $\operatorname{Mg}(\%)$ & 0.0226 & 0.001 & 0.0309 & 0.44 & \\
\hline & $\mathrm{N}(\%)$ & 0.1915 & 0.0579 & 0.2407 & 0.49 & \\
\hline & $\mathrm{P}(\%)$ & 0.0196 & $7.00 \mathrm{E}-04$ & 0.0262 & 0.52 & \\
\hline & $\mathrm{SLA}(\mathrm{m} 2 \mathrm{~g}-1)$ & 0.0012 & 0 & 0.0017 & 0.38 & \\
\hline & Thickness (mm) & 0.0288 & 0.0014 & 0.0379 & 0.55 & \\
\hline \multirow[t]{8}{*}{ Peru } & $\operatorname{Amax}(\mu \mathrm{mol} m-2 \mathrm{~s}-1)$ & 3.0672 & 15.6312 & 3.9536 & 0.25 & \multirow{8}{*}{0.80} \\
\hline & Area $(\mathrm{cm} 2)$ & 90.8151 & 24976.1136 & 158.0383 & 0.38 & \\
\hline & Asat $(\mu \mathrm{mol} m-2 \mathrm{~s}-1)$ & 1.5203 & 3.9208 & 1.9801 & 0.43 & \\
\hline & $\mathrm{C}(\%)$ & 1.4446 & 3.714 & 1.9272 & 0.35 & \\
\hline & $\mathrm{Ca}(\%)$ & 0.1586 & 0.06 & 0.2449 & 0.64 & \\
\hline & Dry mass (g) & 1.4424 & 10.7439 & 3.2778 & 0.26 & \\
\hline & Fresh mass (g) & 1.256 & 3.6084 & 1.8996 & 0.2 & \\
\hline & Leaf water content (\%) & 8.2209 & 138.2141 & 11.7564 & 0.36 & \\
\hline
\end{tabular}




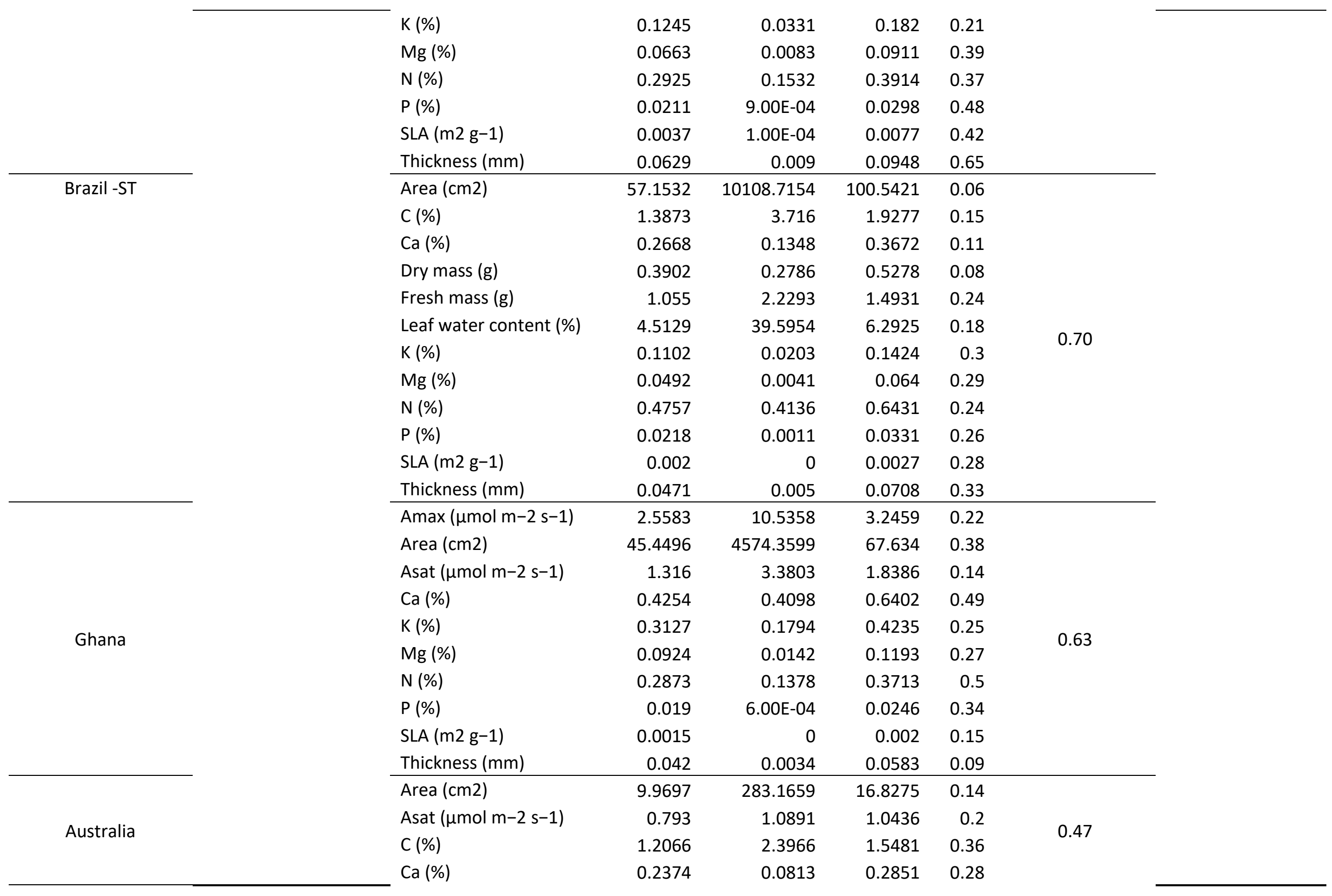




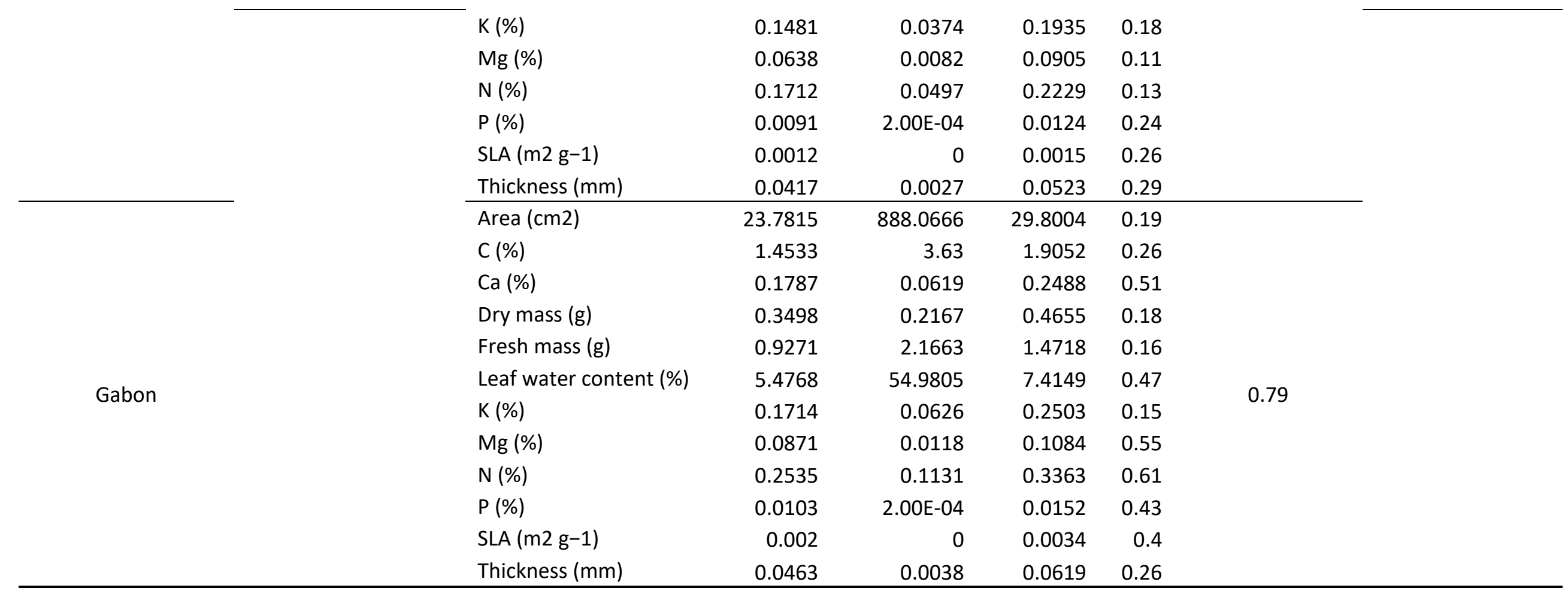

Brazil -NX: Nova Xavantina; Brazil -ST: Santarem. MAE: Mean Absolute Error; MSE: Mean Square Error; RMSE: Root mean square error. 
Table S5. Statistical results on the test data for the models fitted per region for each functional trait.

\begin{tabular}{|c|c|c|c|c|c|}
\hline Locations & Trait & MAE & MSE & RMSE & $\mathbf{R}^{2}$ \\
\hline \multirow{10}{*}{ Australia } & Area $(\mathrm{cm} 2)$ & $7.89 \mathrm{E}+00$ & $1.64 \mathrm{E}+02$ & $1.28 \mathrm{E}+01$ & 0.35 \\
\hline & Asat ( $\mu \mathrm{mol} \mathrm{m}-2 \mathrm{~s}-1)$ & 7.51E-01 & $1.02 \mathrm{E}+00$ & $1.01 \mathrm{E}+00$ & 0.03 \\
\hline & $\mathrm{C}(\%)$ & 9.67E-01 & $1.87 \mathrm{E}+00$ & $1.37 \mathrm{E}+00$ & 0.34 \\
\hline & $\mathrm{Ca}(\%)$ & 1.87E-01 & $5.74 \mathrm{E}-02$ & $2.40 \mathrm{E}-01$ & 0.33 \\
\hline & K (\%) & $1.23 \mathrm{E}-01$ & $3.50 \mathrm{E}-02$ & $1.87 \mathrm{E}-01$ & 0.06 \\
\hline & $\mathrm{Mg}(\%)$ & $6.16 \mathrm{E}-02$ & $1.02 \mathrm{E}-02$ & 1.01E-01 & 0.12 \\
\hline & $N(\%)$ & $1.53 \mathrm{E}-01$ & 4.80E-02 & 2.19E-01 & 0.17 \\
\hline & $\mathrm{P}(\%)$ & 7.60E-03 & $1.00 \mathrm{E}-04$ & 1.07E-02 & 0.21 \\
\hline & SLA (m2 g-1) & $9.00 \mathrm{E}-04$ & $0.00 \mathrm{E}+00$ & $1.30 \mathrm{E}-03$ & 0.25 \\
\hline & Thickness (mm) & 3.47E-02 & $2.30 \mathrm{E}-03$ & 4.77E-02 & 0.21 \\
\hline \multirow{13}{*}{ Gabon } & Area $(\mathrm{cm} 2)$ & $2.97 E+01$ & $2.07 E+03$ & $4.55 E+01$ & 0.11 \\
\hline & $\mathrm{C}(\%)$ & $1.26 \mathrm{E}+00$ & $2.95 E+00$ & $1.72 \mathrm{E}+00$ & 0.22 \\
\hline & $\mathrm{Ca}(\%)$ & $1.55 \mathrm{E}-01$ & $4.51 E-02$ & $2.12 \mathrm{E}-01$ & 0.39 \\
\hline & Dry mass (g) & $5.92 \mathrm{E}-01$ & $1.72 \mathrm{E}+00$ & $1.31 \mathrm{E}+00$ & 0.22 \\
\hline & Fresh mass (g) & $1.06 \mathrm{E}+00$ & $3.39 E+00$ & $1.84 \mathrm{E}+00$ & 0.11 \\
\hline & Leaf water content & & & & \\
\hline & (\%) & $5.05 E+00$ & $4.34 \mathrm{E}+01$ & $6.59 E+00$ & 0.38 \\
\hline & K (\%) & 1.87E-01 & $7.25 \mathrm{E}-02$ & 2.69E-01 & 0.24 \\
\hline & $\mathrm{Mg}(\%)$ & $6.48 \mathrm{E}-02$ & $7.20 \mathrm{E}-03$ & $8.51 \mathrm{E}-02$ & 0.52 \\
\hline & $\mathrm{N}(\%)$ & $2.88 \mathrm{E}-01$ & $1.56 \mathrm{E}-01$ & $3.95 \mathrm{E}-01$ & 0.50 \\
\hline & $\mathrm{P}(\%)$ & $1.14 \mathrm{E}-02$ & $3.00 \mathrm{E}-04$ & $1.70 \mathrm{E}-02$ & 0.60 \\
\hline & SLA (m2 g-1) & $1.90 \mathrm{E}-03$ & $0.00 \mathrm{E}+00$ & $2.80 \mathrm{E}-03$ & 0.15 \\
\hline & Thickness (mm) & 4.70E-02 & $3.80 \mathrm{E}-03$ & $6.14 \mathrm{E}-02$ & 0.23 \\
\hline \multirow{10}{*}{ Ghana } & Amax $(\mu \mathrm{mol} m-2 \mathrm{~s}-1)$ & $2.19 \mathrm{E}+00$ & $7.71 \mathrm{E}+00$ & $2.78 \mathrm{E}+00$ & 0.49 \\
\hline & Area $(\mathrm{cm} 2)$ & $3.73 E+01$ & $2.94 \mathrm{E}+03$ & $5.42 \mathrm{E}+01$ & 0.29 \\
\hline & Asat $(\mu \mathrm{mol} m-2 \mathrm{~s}-1)$ & $1.09 \mathrm{E}+00$ & $1.86 \mathrm{E}+00$ & $1.36 \mathrm{E}+00$ & 0.36 \\
\hline & $\mathrm{Ca}(\%)$ & 3.99E-01 & 5.03E-01 & 7.09E-01 & 0.53 \\
\hline & K (\%) & $2.85 \mathrm{E}-01$ & $1.61 \mathrm{E}-01$ & $4.01 \mathrm{E}-01$ & 0.23 \\
\hline & $\operatorname{Mg}(\%)$ & $9.77 \mathrm{E}-02$ & $1.81 \mathrm{E}-02$ & $1.35 \mathrm{E}-01$ & 0.15 \\
\hline & $\mathrm{N}(\%)$ & 2.77E-01 & 1.57E-01 & $3.96 \mathrm{E}-01$ & 0.52 \\
\hline & $\mathrm{P}(\%)$ & $1.61 \mathrm{E}-02$ & $5.00 \mathrm{E}-04$ & $2.15 \mathrm{E}-02$ & 0.47 \\
\hline & $\mathrm{SLA}(\mathrm{m} 2 \mathrm{~g}-1)$ & $1.30 \mathrm{E}-03$ & $0.00 E+00$ & $1.60 \mathrm{E}-03$ & 0.14 \\
\hline & Thickness $(\mathrm{mm})$ & $2.96 \mathrm{E}-02$ & $1.60 \mathrm{E}-03$ & $4.02 \mathrm{E}-02$ & 0.22 \\
\hline \multirow{12}{*}{ Malaysia } & Amax $(\mu \mathrm{mol} m-2 \mathrm{~s}-1)$ & $3.86 \mathrm{E}+00$ & $2.58 \mathrm{E}+01$ & $5.08 \mathrm{E}+00$ & 0.48 \\
\hline & Area $(\mathrm{cm} 2)$ & $1.33 \mathrm{E}+02$ & $5.64 \mathrm{E}+04$ & $2.38 \mathrm{E}+02$ & 0.11 \\
\hline & Asat $(\mu \mathrm{mol} m-2 \mathrm{~s}-1)$ & $1.70 E+00$ & $4.55 E+00$ & $2.13 E+00$ & 0.36 \\
\hline & $C(\%)$ & $1.57 \mathrm{E}+00$ & $4.78 \mathrm{E}+00$ & $2.19 E+00$ & 0.38 \\
\hline & $\mathrm{Ca}(\%)$ & 2.96E-01 & $2.78 \mathrm{E}-01$ & 5.27E-01 & 0.50 \\
\hline & Dry mass (g) & $1.57 \mathrm{E}+00$ & $9.55 E+00$ & $3.09 E+00$ & 0.07 \\
\hline & Fresh mass (g) & $4.03 E+00$ & $7.70 \mathrm{E}+01$ & $8.77 E+00$ & 0.03 \\
\hline & $\begin{array}{l}\text { Leaf water content } \\
\text { (\%) }\end{array}$ & $3.89 \mathrm{E}+00$ & $2.85 \mathrm{E}+01$ & $5.34 \mathrm{E}+00$ & 0.24 \\
\hline & K (\%) & 2.96E-01 & $1.90 \mathrm{E}-01$ & 4.35E-01 & 0.28 \\
\hline & $\mathrm{Mg}(\%)$ & $9.84 \mathrm{E}-02$ & $1.86 \mathrm{E}-02$ & $1.36 \mathrm{E}-01$ & 0.31 \\
\hline & $\mathrm{N}(\%)$ & 2.27E-01 & $1.29 \mathrm{E}-01$ & $3.58 \mathrm{E}-01$ & 0.27 \\
\hline & $\mathrm{P}(\%)$ & $2.08 \mathrm{E}-02$ & 1.30E-03 & $3.64 \mathrm{E}-02$ & 0.34 \\
\hline
\end{tabular}




\begin{tabular}{|c|c|c|c|c|c|}
\hline & SLA (m2g-1) & $1.70 \mathrm{E}-03$ & $0.00 E+00$ & $2.70 \mathrm{E}-03$ & 0.06 \\
\hline & Thickness (mm) & 5.05E-02 & 8.40E-03 & $9.16 \mathrm{E}-02$ & 0.31 \\
\hline \multirow{13}{*}{ Brazil -NX } & Amax $(\mu \mathrm{mol} m-2 \mathrm{~s}-1)$ & $2.40 \mathrm{E}+00$ & $1.06 \mathrm{E}+01$ & $3.26 \mathrm{E}+00$ & 0.42 \\
\hline & Area $(\mathrm{cm} 2)$ & $1.54 \mathrm{E}+01$ & $4.50 \mathrm{E}+02$ & $2.12 \mathrm{E}+01$ & 0.08 \\
\hline & Asat $(\mu \mathrm{mol} m-2 \mathrm{~s}-1)$ & $1.48 \mathrm{E}+00$ & $3.60 \mathrm{E}+00$ & $1.90 \mathrm{E}+00$ & 0.52 \\
\hline & $\mathrm{Ca}(\%)$ & $5.70 \mathrm{E}-02$ & $5.10 \mathrm{E}-03$ & 7.15E-02 & 0.49 \\
\hline & Dry mass (g) & 2.12E-01 & $1.16 \mathrm{E}-01$ & $3.40 \mathrm{E}-01$ & 0.38 \\
\hline & Fresh mass (g) & $5.42 \mathrm{E}-01$ & 8.21E-01 & 9.06E-01 & 0.31 \\
\hline & $\begin{array}{l}\text { Leaf water content } \\
\text { (\%) }\end{array}$ & $2.91 E+00$ & $1.50 \mathrm{E}+01$ & $3.88 \mathrm{E}+00$ & 0.07 \\
\hline & $\mathrm{K}(\%)$ & $5.81 \mathrm{E}-02$ & $6.00 \mathrm{E}-03$ & 7.74E-02 & 0.07 \\
\hline & $\mathrm{Mg}(\%)$ & $2.32 \mathrm{E}-02$ & 9.00E-04 & 3.05E-02 & 0.46 \\
\hline & N (\%) & 1.63E-01 & 4.22E-02 & 2.05E-01 & 0.52 \\
\hline & $\mathrm{P}(\%)$ & 1.49E-02 & 4.00E-04 & 1.89E-02 & 0.68 \\
\hline & SLA (m2 g-1) & 8.00E-04 & $0.00 \mathrm{E}+00$ & $1.10 \mathrm{E}-03$ & 0.54 \\
\hline & Thickness (mm) & $2.26 \mathrm{E}-02$ & $9.00 \mathrm{E}-04$ & $3.00 \mathrm{E}-02$ & 0.66 \\
\hline \multirow{14}{*}{ Peru } & $\operatorname{Amax}(\mu \mathrm{mol} m-2 \mathrm{~s}-1)$ & $2.43 \mathrm{E}+00$ & $9.28 \mathrm{E}+00$ & $3.05 E+00$ & 0.36 \\
\hline & Area $(\mathrm{cm} 2)$ & $1.10 E+02$ & $5.90 \mathrm{E}+04$ & $2.43 \mathrm{E}+02$ & 0.18 \\
\hline & Asat $(\mu \mathrm{mol} m-2 \mathrm{~s}-1)$ & $1.36 \mathrm{E}+00$ & $3.17 \mathrm{E}+00$ & $1.78 \mathrm{E}+00$ & 0.38 \\
\hline & $C(\%)$ & $1.27 \mathrm{E}+00$ & $3.19 \mathrm{E}+00$ & $1.79 \mathrm{E}+00$ & 0.47 \\
\hline & $\mathrm{Ca}(\%)$ & $1.25 \mathrm{E}-01$ & $3.41 \mathrm{E}-02$ & $1.85 \mathrm{E}-01$ & 0.69 \\
\hline & Dry mass (g) & $1.53 \mathrm{E}+00$ & $1.09 \mathrm{E}+01$ & $3.30 E+00$ & 0.09 \\
\hline & Fresh mass (g) & $1.01 E+00$ & $1.97 \mathrm{E}+00$ & $1.40 \mathrm{E}+00$ & 0.2 \\
\hline & $\begin{array}{l}\text { Leaf water content } \\
\text { (\%) }\end{array}$ & $8.07 E+00$ & $1.29 \mathrm{E}+02$ & $1.13 \mathrm{E}+01$ & 0.34 \\
\hline & $\mathrm{K}(\%)$ & $1.03 \mathrm{E}-01$ & $1.74 \mathrm{E}-02$ & $1.32 \mathrm{E}-01$ & 0.3 \\
\hline & $\mathrm{Mg}(\%)$ & $5.52 \mathrm{E}-02$ & $6.20 \mathrm{E}-03$ & 7.89E-02 & 0.46 \\
\hline & $N(\%)$ & $2.52 \mathrm{E}-01$ & 1.14E-01 & 3.37E-01 & 0.44 \\
\hline & $\mathrm{P}(\%)$ & $2.18 \mathrm{E}-02$ & 8.00E-04 & $2.89 \mathrm{E}-02$ & 0.49 \\
\hline & $\mathrm{SLA}(\mathrm{m} 2 \mathrm{~g}-1)$ & $2.70 \mathrm{E}-03$ & $0.00 \mathrm{E}+00$ & $5.10 \mathrm{E}-03$ & 0.32 \\
\hline & Thickness (mm) & $5.66 \mathrm{E}-02$ & $9.10 \mathrm{E}-03$ & $9.53 \mathrm{E}-02$ & 0.64 \\
\hline \multirow{12}{*}{ Brazil -ST } & Area $(\mathrm{cm} 2)$ & $4.31 E+01$ & $5.68 \mathrm{E}+03$ & $7.53 \mathrm{E}+01$ & 0.04 \\
\hline & $\mathrm{C}(\%)$ & $1.36 \mathrm{E}+00$ & $3.72 \mathrm{E}+00$ & $1.93 \mathrm{E}+00$ & 0.07 \\
\hline & $\mathrm{Ca}(\%)$ & 2.36E-01 & 8.75E-02 & 2.96E-01 & 0.15 \\
\hline & Dry mass (g) & $3.86 \mathrm{E}-01$ & $3.18 \mathrm{E}-01$ & 5.64E-01 & 0.25 \\
\hline & Fresh mass (g) & $1.07 E+00$ & $3.39 E+00$ & $1.84 \mathrm{E}+00$ & 0.18 \\
\hline & $\begin{array}{l}\text { Leaf water content } \\
\text { (\%) }\end{array}$ & $4.22 \mathrm{E}+00$ & $3.48 \mathrm{E}+01$ & $5.90 \mathrm{E}+00$ & 0.05 \\
\hline & K (\%) & $1.08 \mathrm{E}-01$ & $2.32 \mathrm{E}-02$ & $1.52 \mathrm{E}-01$ & 0.29 \\
\hline & $\mathrm{Mg}(\%)$ & 4.95E-02 & 4.00E-03 & $6.32 \mathrm{E}-02$ & 0.28 \\
\hline & $\mathrm{N}(\%)$ & $3.26 \mathrm{E}-01$ & $1.82 \mathrm{E}-01$ & 4.27E-01 & 0.30 \\
\hline & $\mathrm{P}(\%)$ & $2.06 \mathrm{E}-02$ & $1.00 \mathrm{E}-03$ & 3.17E-02 & 0.47 \\
\hline & $\mathrm{SLA}(\mathrm{m} 2 \mathrm{~g}-1)$ & $1.80 \mathrm{E}-03$ & $0.00 \mathrm{E}+00$ & $2.20 \mathrm{E}-03$ & 0.29 \\
\hline & Thickness (mm) & 4.37E-02 & 4.40E-03 & $6.62 \mathrm{E}-02$ & 0.42 \\
\hline
\end{tabular}

Brazil -NX: Nova Xavantina; Brazil -ST: Santarem. MAE: Mean Absolute Error; MSE: Mean Square Error; RMSE: Root mean square error. 
Neyret, M., Bentley, L.P., Oliveras, I., Marimon, B.S., Marimon-Junior, B.H., Almeida de Oliveira, E., et al. (2016). Examining variation in the leaf mass per area of dominant species across two contrasting tropical gradients in light of community assembly. Ecology and evolution, 6, 5674-5689. 